\title{
Factors Affecting Heat Transport in an Ocean General Circulation Model
}

\author{
Igor Kamenkovich, * Jochem Marotzke, and Peter H. Stone \\ Center for Global Change Science, Massachusetts Institute of Technology, Cambridge, Massachusetts
}

(Manuscript received 21 July 1998, in final form 2 April 1999)

\begin{abstract}
A global ocean general circulation model with idealized geometry and coupled to a simple representation of atmospheric energy fluxes is used to investigate which physical factors determine meridional heat transport. A particular focus is on causes for the common underestimation of heat transport in ocean general circulation models. The model is also forced by an idealized wind stress and moisture flux profiles.

The zonal average of surface heat flux is obtained from a simple radiation parameterization and the divergence of observed atmospheric heat transport. In addition, zonal mixing in the atmosphere is implied by the relaxation of the sea surface temperature (SST) to its zonal average. A finite relaxation timescale results in a substantial increase in the meridional mass overturning in the "Atlantic" basin compared to the case with "infinitely efficient" zonal atmospheric mixing, owing to the admittance of zonal SST gradients. However, heat transport changes only by a small amount. When atmospheric zonal mixing is changed to interbasin mixing, meridional heat transport increases significantly. Doubling the width of the Pacific basin leads to a large increase in the Pacific heat transport, induced by both the meridional overturning and the horizontal gyre circulations.

If the horizontal viscosity is decreased and the zonal resolution is increased near the boundaries, the resulting larger speed of the western boundary currents causes a noticable increase in the Atlantic basin's heat transport.

The introduction of the Gent-McWilliams eddy parameterization leads to a substantial decrease in the strength of the overturning circulation in the Atlantic basin, presumably because the overall amount of diapycnal mixing is reduced. However, the decrease in the heat transport is much smaller because the thermocline is sharper and the deep ocean colder, resulting in enhanced vertical temperature contrast. Apparent disagreements with and among previous results are explained through the different effects of diapycnal mixing in the North Atlantic and elsewhere in the model.
\end{abstract}

\section{Introduction}

Numerical ocean general circulation models (GCM) have proved to be very useful for understanding different physical aspects of the thermohaline circulation. However, the idealization of many physical processes in numerical models often leads to errors in simulated physical fields. One of the common problems that many oceanic models share is the underestimated meridional heat transport (e.g., Böning et al. 1995). Unrealistic oceanic heat transport, apart from indicating missing physics, is one of the main reasons for a drift of coupled atmosphere-ocean models from the present climate (Marotzke and Stone 1995; Weaver and Hughes 1996).

The understanding of the reasons for such underestimation is crucial for an improvement of our ability to

\footnotetext{
* Current affiliation: Joint Institute for the Study of the Atmosphere and the Oceans, University of Washington, Seattle, Washington.

Corresponding author address: Joint Institute for the Study of the Atmosphere and the Oceans, Box 354235, University of Washington, Seattle, WA 98195.
}

model ocean circulation but is generally difficult in complex GCMs. For this reason, idealized models with simplified dynamics are widely used. These models try to isolate some physical processes either by leaving out or by parameterizing others. The hope is that such an idealized model can capture dynamical properties of more advanced models and at the same time remain simple enough for detailed understanding. Recently, significant progress has been made in understanding the dynamics of the thermohaline circulation (THC) in a single-basin model (Marotzke 1997; Klinger and Marotzke 1999; Marotzke and Klinger 1999, manuscript submitted to $J$. Phys. Oceanogr, hereafter MK). However, a higher level of complexity is needed for even a schematic representation of the "conveyor belt" circulation, in which waters sink in the North Atlantic and upwell in the Pacific and Indian Oceans (Gordon 1986).

In the present study, we will utilize a two-basin model with highly idealized geometry. The degree of geometric complexity is minimal for the representation of the conveyor belt circulation (Marotzke and Willebrand 1991). The model is forced with surface boundary conditions that are identical in both model basins. The only longitudinal dependence enters through the dependence 
of the surface heat flux on surface temperature. The model therefore represents the next step from the family of single-basin models toward GCMs with realistic geometry. We consider a number of physical factors that are expected to have a large effect on the THC and heat transport in the model. Below, we will list them together with a brief discussion of why we consider them important and a summary of related studies.

Surface boundary conditions are among the most important factors controlling the THC in an ocean GCM. They reflect implicit assumptions about atmospheric transports and hence ocean-atmosphere interactions. We choose the boundary conditions (see section 2) to be very similar to those used by Wang et al. (1999, WSM hereafter), in which the surface heat flux is calculated from modeled sea surface temperature (SST) and observed atmospheric meridional heat transport (not atmospheric temperature). One effect of the atmosphere on the ocean is the zonal transfer of heat by zonal winds from one ocean basin to another. This process has not been addressed much in the modeling literature (see, however, Mikolajewicz et al. 1997), although it has a large effect on the zonal structure of surface temperature. Zonal winds tend to homogenize temperature within each basin; in addition, they provide an interbasin heat flux. In this study we will consider the impact of parameterizing these two effects on heat transport and the THC (experiments ZONAV, ATMIX, and ATMIXB).

The influence of horizontal resolution on the poleward heat transport was studied by Wang et al. (1995) and Fanning and Weaver (1997). These authors used single-hemisphere models for sets of experiments with different horizontal resolution and horizontal viscosity and found an increase in the poleward heat transport with increased resolution, owing to the larger amount of heat carried by the baroclinic gyres. Related to this, Wunsch (1996) suggested that proper resolution of western boundary currents is more important for successful modeling of large-scale circulation than fine resolution of the oceanic interior.

We address the roles of horizontal resolution and speed of boundary currents in experiment FAST, in which the zonal grid spacing in the western boundary region is decreased compared to the interior of an ocean basin. In addition, we decrease the horizontal viscosity, which results in faster boundary currents, and we study the overall effect on the heat transport.

One of the biggest problems in the modeling of ocean circulation on climatic timescales is that it is difficult to adequately resolve mesoscale eddies, the effects of which must be parameterized instead. The oldest approach is to assume downgradient diffusion of momentum and tracers in the horizontal and vertical directions, with constant diffusion coefficients. Gent and McWilliams (1990) introduced a scheme that combines isopycnal diffusion with eddy-induced quasi-advective transports in the equations for tracers. The scheme has been used in a number of studies but published results appear contradictory. Danabasoglu et al. (1994) concluded that the introduction of the scheme improved both the representation of the THC and the heat transport in their model. In particular, the authors reported cancellation of the Deacon cell as a tracer transport mechanism in the Antarctic Circumpolar Current (ACC), a sharper main thermocline, and a cooler abyssal ocean. The scheme was also shown to eliminate spurious upwelling in the regions of western boundary currents (Böning et al. 1995); the result was an increase in the overturning strength and the associated heat transport.

In contrast, Duffy et al. (1997), Weaver and Eby (1997), and Jiang et al. (1999, manuscript submitted to J. Phys. Oceanogr.) reported that the THC was significantly weakened when the Gent-McWilliams (GM hereafter) scheme was used. The authors confirmed cooling of the deep ocean as an effect of the scheme, which, by itself, increases northward heat transport in the Atlantic, where cold deep waters flow southward. However, a weakening of the THC counteracts the cooling of the deep ocean in increasing the heat transport. Experiment GM investigates the overall effect of the GM scheme on the heat transport in our model. In particular, we will focus on the effects of (spurious) horizontal diapycnal mixing. Bryan (1987) reported that the THC increased with increasing vertical diffusion coefficient in his single-basin model. The dependence is related to the deepening of the isopycnal surfaces and resulting stronger horizontal density gradients. Gerdes et al. (1991) emphasized that the diapycnal, rather than vertical diffusivity, was the important factor controlling the strength of THC. Marotzke and Klinger (MK) investigated the effect of the horizontal distribution of diapycnal mixing on THC strength, in particular the cross-equatorial transport. Their results will help us resolve the apparently contradictory results found in earlier studies.

Another physical factor that is expected to have a large effect on heat transport is the basin geometry. The widths of both Atlantic and Pacific basins by WSM were chosen to be the same. But Wang et al. (1995) and Klinger (1996) demonstrated that the heat transport in a barotropic, single-basin model strongly depends on the width of the basin. The generalization to a GCM would imply increases in gyre heat transport with basin width. We will look at the role of the width of the "Pacific" basin (defined as the one without northern deep-water formation) in experiment WIDE.

The paper is organized as follows. In section 2 we give the description of the model. Results from five different experiments are given in section 3 . In those experiments several changes to the original model setup (experiment ZONAV) will be made one at a time. In each experiment we change one aspect of the preceding setup and analyze the changes. A summary and discussion of the results are given in section 4 . 


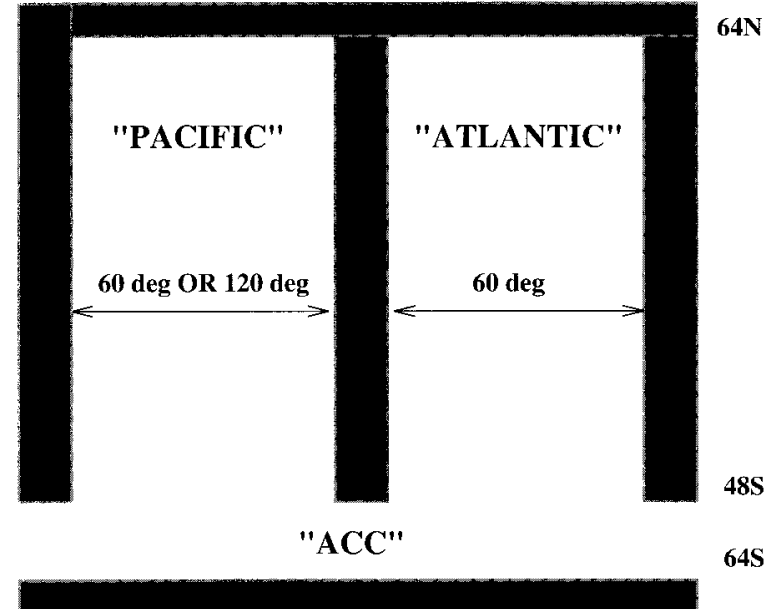

FIG. 1. Geometry of the model.

\section{Description of the model}

The primitive equation, hydrostatic model developed at GFDL, Princeton (MOM2), is used for our experiments. The model geometry is very similar to that used by Marotzke and Willebrand (1991) (see Fig. 1). It consists of two rectangular basins extending from $64^{\circ} \mathrm{N}$ to $48^{\circ} \mathrm{S}$. The basins are connected by a channel extending from $48^{\circ}$ to $64^{\circ} \mathrm{S}$, representing the ACC. We will concentrate on "conveyor belt" equilibria only, and, therefore, "Atlantic" will denote the basin with deep sinking in the north, and "Pacific" will stand for the other basin. Both basins have the same width of $60^{\circ}$ in the experiments ZONAV, ATMIXB, FAST, and GM. The width of the Pacific basin is doubled to $120^{\circ}$ in experiment WIDE. Note the absence of an Indian Ocean in the model, in which the Pacific basin will therefore play the role of both Pacific and Indian Oceans in the Southern Hemisphere heat budget.

The horizontal resolution is $4^{\circ}$ in latitude and either constant $3.75^{\circ}$, or varying from $1.75^{\circ}$ to $3.75^{\circ}$ in longitude (see Table 1). The model has 15 layers in the vertical with thicknesses increasing downward from 50 to $500 \mathrm{~m}$. The bottom is flat. No-slip conditions for velocity ( $\mathbf{v}=0$, where $\mathbf{v}$ is horizontal velocity) are applied at all lateral walls. Free-slip conditions are used $(d \mathbf{v} / d z=0)$ at the bottom, except in the ACC, which is treated differently than in WSM. Instead of specifying its transport at the southern boundary, a bottom drag is applied:

$$
D_{\text {bottom }}=c_{\text {bot }}|\mathbf{v}| \mathbf{v} \text {. }
$$

The drag is zero everywhere outside the ACC region. Both large momentum viscosity (see Table 1) and bottom friction then balance the wind stress torque in the ACC. As a result, the ACC's transport is typically between 140 and $180 \mathrm{~Sv}\left(\mathrm{~Sv} \equiv 10^{6} \mathrm{~m}^{3} \mathrm{~s}^{-1}\right)$ in all experiments. Although somewhat larger than the value of approximately $125 \mathrm{~Sv}$ from the direct measurements (Whitworth and Peterson 1985), it is a reasonable number for a coarse-resolution model with no topography.

Boundary conditions for tracers are insulating at the lateral walls and bottom. In experiments ZONAV, ATMIXB, and FAST, horizontal and vertical diffusion is used for tracers. The Gent-McWilliams scheme (Gent and McWilliams 1990) is used in experiments GM and WIDE. The choices for mixing coefficients are given in Table 1.

We use asynchronous integration [unequal time steps for tracer and momentum equations, Bryan (1984)] to accelerate convergence to equilibrium, which is achieved in each case. The integrations start from initial conditions of no motion, constant salinity, and horizontally averaged profiles from Levitus (1982) for temperature in experiments ZONAV and WIDE. Experiments ATMIX, FAST, and GM are started subsequently one after another, each providing initial conditions for the following experiment and thus saving computer time.

Surface boundary conditions for momentum are the same as in Marotzke and Willebrand (1991) and are given by a specified zonal wind stress, which is a function of latitude only. The idealized profile is symmetric around the equator but otherwise chosen to resemble data; see Fig. 2.

Surface boundary conditions for tracers are very similar to those used in WSM, so we will only provide a brief description. The surface heat flux consists of four terms:

$$
F_{H}=\left[-\frac{\partial}{\partial y} H_{t}-(\mathrm{LW}-\mathrm{SW})\right] / \epsilon+M_{\mathrm{zon}} .
$$

\begin{tabular}{|c|c|c|c|}
\hline Parameters & ZONAV, ATMIX B experiments & FAST experiment & GM, WIDE experiments \\
\hline Zonal resolution $\Delta \tau$ & $3.75^{\circ}$ & from $1.75^{\circ}$ to $3.75^{\circ}$ & from $1.75^{\circ}$ to $3.75^{\circ}$ \\
\hline Meridional resolution $\Delta \lambda$ & $4^{\circ}$ & $4^{\circ}$ & $4^{\circ}$ \\
\hline Vertical viscosity $A_{v}$ & $0.01 \mathrm{~m}^{2} \mathrm{~s}^{-1}$ & $0.01 \mathrm{~m}^{2} \mathrm{~s}^{-1}$ & $0.01 \mathrm{~m}^{2} \mathrm{~s}^{-1}$ \\
\hline Horizontal viscosity $A_{h}$ & $2.5 \times 10^{5} \mathrm{~m}^{2} \mathrm{~s}^{-1}$ & $0.5 \times 10^{5} \mathrm{~m}^{2} \mathrm{~s}^{-1}$ & $0.5 \times 10^{5} \mathrm{~m}^{2} \mathrm{~s}^{-1}$ \\
\hline Vertical diffusivity $\kappa_{v}$ & $5 \times 10^{-5} \mathrm{~m}^{2} \mathrm{~s}^{-1}$ & $5 \times 10^{-5} \mathrm{~m}^{2} \mathrm{~s}^{-1}$ & $5 \times 10^{-5} \mathrm{~m}^{2} \mathrm{~s}^{-1}$ \\
\hline Horizontal diffusivity $\kappa_{h}$ & $10^{3} \mathrm{~m}^{2} \mathrm{~s}^{-1}$ & $10^{3} \mathrm{~m}^{2} \mathrm{~s}^{-1}$ & 0 \\
\hline Isopycnal diffusivity $\kappa_{I}$ & 0 & 0 & $10^{3} \mathrm{~m}^{2} \mathrm{~s}^{-1}$ \\
\hline Thickness diffusivity $\kappa_{\tau}$ & 0 & 0 & $10^{3} \mathrm{~m}^{2} \mathrm{~s}^{-1}$ \\
\hline Bottom drag coefficient $c_{\text {bot }}$ & 0.25 & 0.5 & 0.5 \\
\hline
\end{tabular}

TABLE 1. Parameters used in the experiments. 


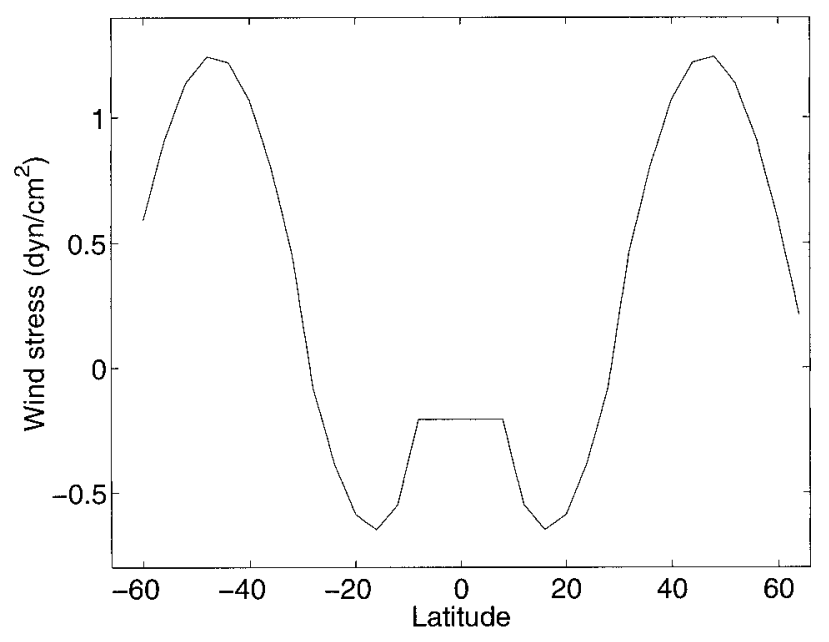

FIG. 2. Idealized wind stress profile used in the model as a function of latitude. Units are dyn $\mathrm{cm}^{-2}$.

The first term in square brackets is the divergence of heat transport in the atmosphere, distributed evenly over a latitude circle $\left(\partial H_{t} / \partial y\right.$, Fig. $\left.3 \mathrm{~b}\right)$; it accounts for both the sensible and latent heat fluxes. The second term represents the radiative balance at the top of the atmosphere (longwave minus shortwave radiation, LW SW), evaluated locally. Both terms are divided by $\epsilon$, the ratio of ocean area to total area at any latitude (see below and Marotzke and Stone 1995). The term $M_{\text {zon }}$ is a zonal redistribution term in the atmosphere and has zero zonal mean. Its precise form varies between experiments and is explained later.

The incoming shortwave radiation SW is fixed (Fig. $3 a)$ and represents the product of solar radiation and (1 $-\alpha$ ), where $\alpha$ is planetary albedo. The outgoing longwave radiation LW is parameterized as a linear function of sea surface temperature $T_{s}$, as in WSM:

$$
\mathrm{LW}=195+2.78 T_{s} .
$$

Equation (2) implies that the atmosphere is in energy balance with the underlying ocean, and the air-sea flux $F_{H}$ is the residual of the atmospheric budget.

Since land masses are represented by narrow strips in the model (see Fig. 1), the effects of land must be parameterized. As in WSM, we assume zero heat capacity of the land and that air temperature over land is equal to the zonal mean SST (needed in the LW term). Therefore, the residue between radiative fluxes and divergence of the atmospheric transport [square bracket in Eq. (2)] over the land must enter the ocean. As a result, we multiply the zonal-mean portion of $F_{H}$ by $1 / \epsilon$, the inverse of the fraction of a latitude circle that is occupied by the oceans in our model. The factor is 3.0 for experiments ZONAV to GM and 2.0 for experiment WIDE.

The moisture flux, which is prescribed from observations, is the same as that used in WSM's control run (Fig. 4). It is balanced separately in the North Atlantic,
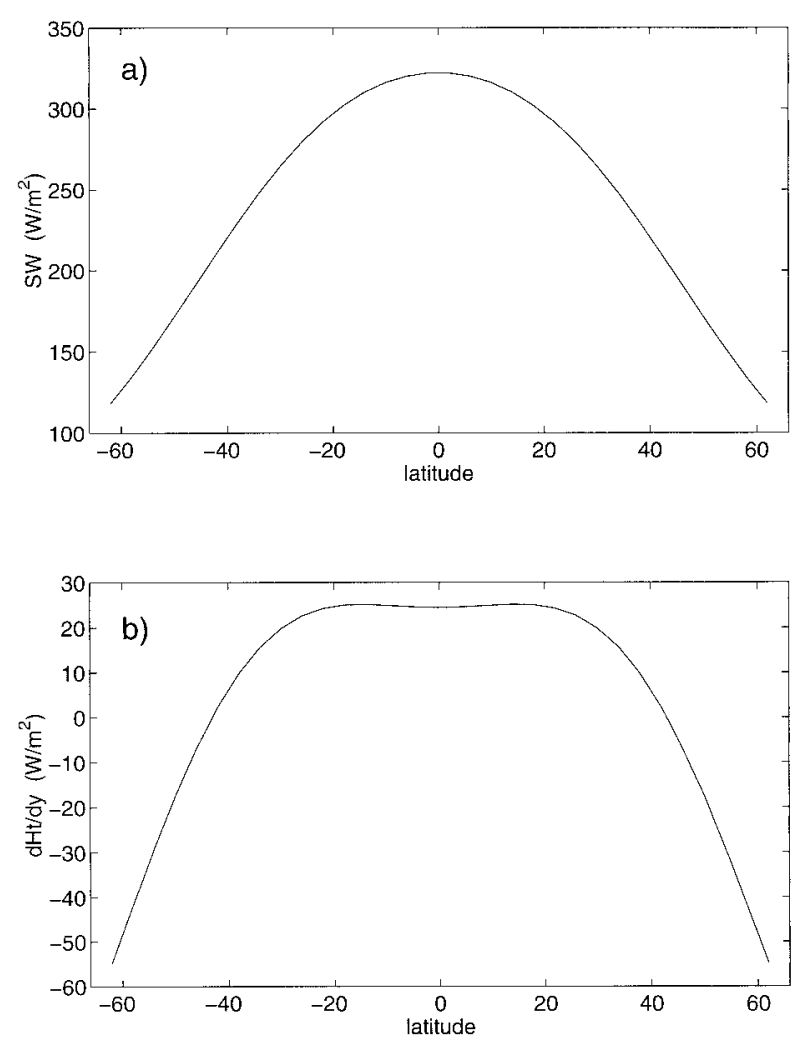

FIG. 3. Components of the surface heat flux: (a) shortwave radiation through the top of the atmosphere and (b) the divergence of atmospheric heat transport. See the text for details. Units are watts per square meter.

North Pacific, South Atlantic, and South Pacific (the latter two including the ACC). The thermohaline boundary conditions are of mixed type, since the zonal-mean heat flux formulation [the first term on the right-hand side of Eq. (2)] is identical to a restoring boundary condition for temperature, as shown by WSM. The re-

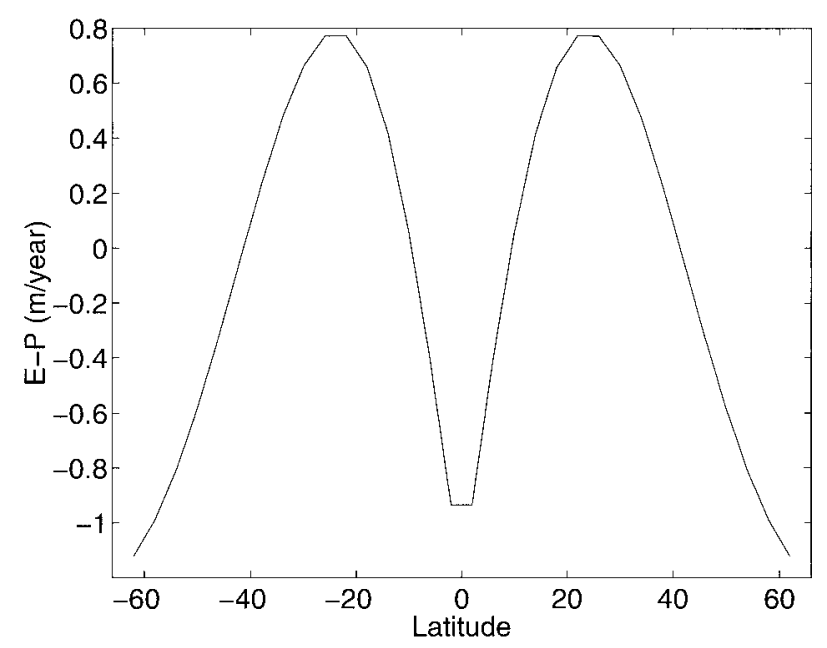

FIG. 4. Moisture flux in the model. Units are meters per year. 
storing timescale is 288 days for the zonal average heat flux, which is considerably longer than the Haney timescale (Haney 1971), which would be approximately 70 days in our case. We will later introduce a much shorter restoring timescale for the law governing deviations from the zonal-mean heat flux, $M_{\text {zon }}$.

Only conveyor belt equilibria with water sinking in the North Atlantic and upwelling in the Pacific are considered here. Other possible equilibria (see, e.g., Marotzke and Willebrand 1991) are avoided by applying an anomalous freshwater flux in the northern parts of both basins during the initial stage of development. A constant value is added to the freshwater flux in the Pacific and subtracted in the Atlantic. The fluxes are made identical in both basins as soon as the conveyor belt circulation is fully established (see WSM), and the integration is continued to equilibrium.

\section{Heat transport components}

The main focus of our analysis is on the northward oceanic heat transport. As usual, we divide the heat transport into several components, which represent different controlling physical mechanisms. The diffusive component is proportional to the zonally and vertically averaged meridional temperature gradients if the horizontal diffusion scheme is used. In the case of isopycnal diffusion (experiments GM and WIDE), the diffusive component is defined as the horizontal component of the diffusive transport along the isopycnal surfaces and depends on the orientation of these surfaces as well as on the temperature distribution.

By decomposing both meridional velocity $v$ and temperature $T$ into zonally averaged parts $\langle v\rangle$ and $\langle T\rangle$ and the residues $v^{\prime}$ and $T^{\prime}$, we can write the advective transport as

$$
\begin{aligned}
\rho C_{p} L \int_{0}^{D}\langle v T\rangle d z= & \rho C_{p} L \int_{0}^{D}\langle v\rangle\langle T\rangle d z \\
& +\rho C_{p} L \int_{0}^{D}\left\langle v^{\prime} T^{\prime}\right\rangle d z
\end{aligned}
$$

where $L$ is the width of a basin, $D$ is its depth, and $C_{p}$ is the specific heat.

The first term on the right-hand side represents the transfer of heat by zonally averaged velocities (overturning component), which scales as the strength of the meridional overturning of mass multiplied by the vertical temperature contrast. The second term on the righthand side of Eq. (4) is the "gyre" component; it owes its existence to a number of mechanisms. To see that, we now decompose each factor in the second term into its depth-averaged part (denoted by an overbar) and the residue (denoted by an asterisk):

$$
\begin{aligned}
\rho C_{p} L \int_{0}^{D}\left\langle v^{\prime} T^{\prime}\right\rangle d z= & \rho C_{p} L D\left\langle\overline{v^{\prime}} \overline{T^{\prime}}\right\rangle \\
& +\rho C_{p} L D\left\langle\overline{\left(v^{\prime}\right)^{*}\left(T^{\prime}\right)^{*}}\right\rangle .
\end{aligned}
$$

The first and second terms in Eq. (5) will be called "barotropic" and "baroclinic" gyre components, respectively; note that $\left(v^{\prime}\right)^{*}$ and $\left(T^{\prime}\right)^{*}$ are three-dimensional quantities.

\section{Results of the experiments}

In this section we describe and analyze a set of experiments aimed at understanding the factors controlling heat transport in the model. The configuration of each experiment will be identical to the preceding one except for a specific addition.

\section{a. Zonally averaged SST: Experiment ZONAV}

The setup of this experiment is largely identical to that in the control run of WSM. As discussed in the introduction, the homogenization of SST by atmospheric zonal winds has a large effect on the surface density and, possibly, on the THC. Because of the lack of a convincing theory for this process, we choose to parameterize it. Similar to WSM, SST is substituted by its zonal average (each basin separately) at each time step. Zonal averaging is an extreme form of the parameterization, implying "infinitely efficient" zonal mixing, which completely eliminates zonal gradients of SST within each basin.

The integration procedure is similar to that in WSM. The model is integrated for 1000 years with restoring conditions for surface salinity and then for another 250 years with a fixed moisture flux. An anomalous moisture flux of $0.95 \mathrm{~m} \mathrm{yr}^{-1}$ is then applied (out of the North Atlantic, into the North Pacific) to establish the conveyor belt circulation. The amplitude of the anomalous flux is then halved after 3500 years and then further reduced by a factor of 4 after another 1500 years and the integration is continued for 1000 years. The anomalous flux is then finally removed, and the integration continued for 3000 years to obtain an equilibrium with freshwater forcing identical over the Atlantic and Pacific. The total length of integration is 9250 years. ${ }^{1}$

\footnotetext{
${ }^{1}$ The results are very similar to those in WSM. The largest difference is in the sea surface salinity, which is slightly lower in our case. The reasons for this difference are not obvious. However, a different version of the GFDL model is used in this study, namely, MOM2 versus MOM1 in WSM; moreover, the treatment of the ACC is different. Sea surface temperatures are nearly identical in both cases.

The difference in surface density leads to somewhat different overturning in the North Atlantic (Fig. 5); the maximum rate of NADW formation is $24 \mathrm{~Sv}$ compared to the value of $21 \mathrm{~Sv}$ in WSM. Weak counterclockwise circulation near the northern boundary in the Atlantic can be seen in Fig. 5. The thermohaline circulation is practically identical in the Pacific, between WSM and here.
} 
a) Meridional overturning in the Pacific (Sv)

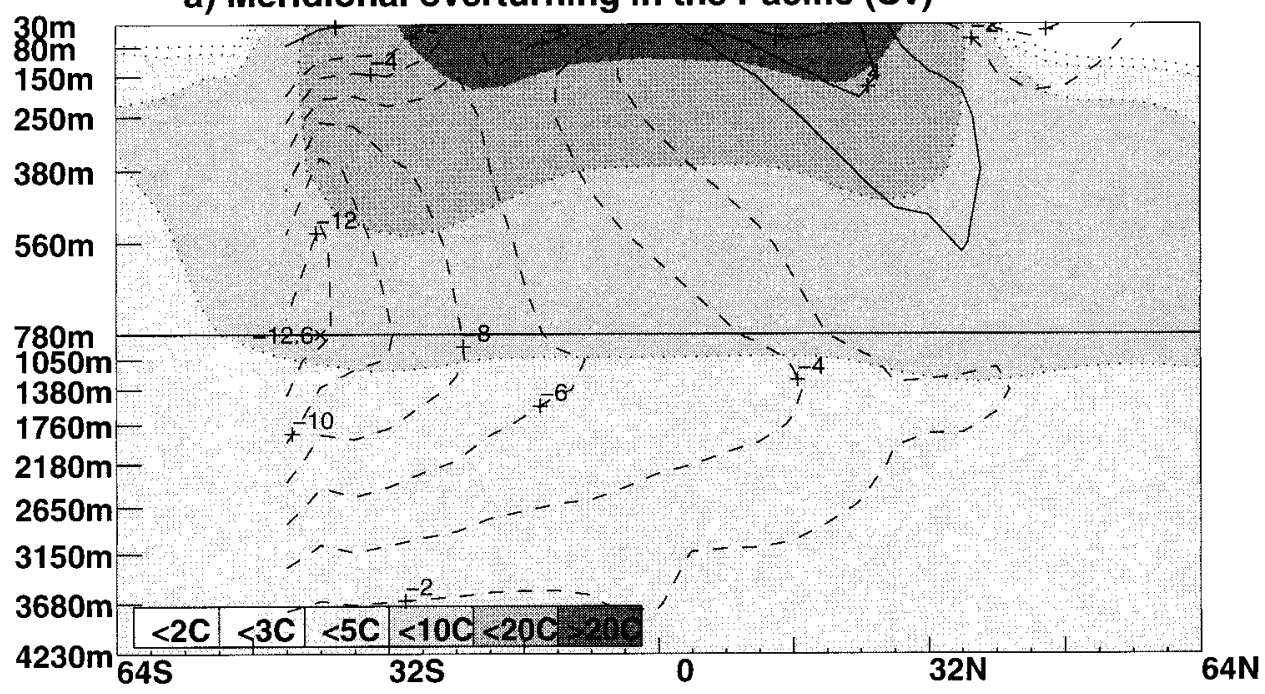

b) Meridional overturning in the Atlantic (Sv)

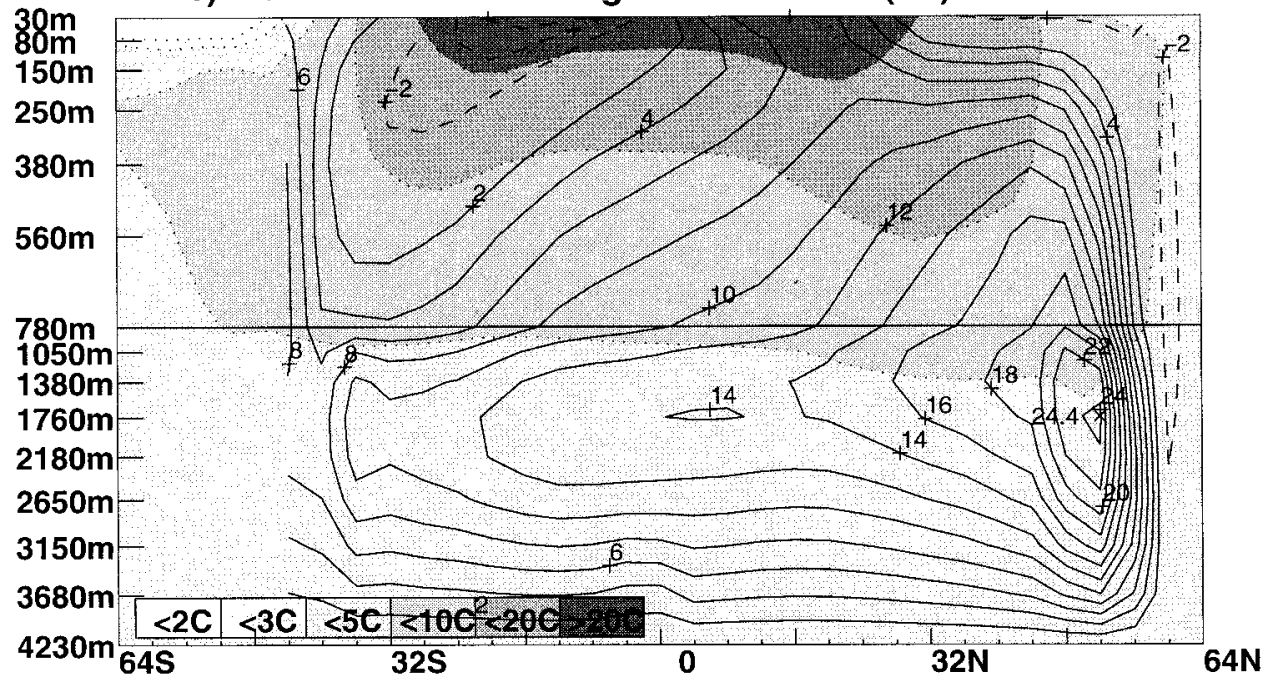

FIG. 5. Meridional overturning streamfunction (Sv) in experiment ZONAV. For (a) the Pacific and (b) the Atlantic; the ACC (south of $48^{\circ} \mathrm{S}$ ) is not included. The solid lines show clockwise circulation; the dashed lines show counterclockwise circulation. Contour interval is $2 \mathrm{~Sv}$. The contours with shading represent zonally averaged temperatures; corresponding intervals are given in the plot.

As can be seen from the schematic representation of the vertical temperature structure in Fig. 5, the deep ocean is very warm in the experiment, between $3^{\circ}$ and $5^{\circ}$ in either basin. Low vertical temperature contrast makes it difficult for the thermohaline circulation to transport a large amount of heat. The resulting northward heat transport is shown in Fig. 6. The peak value in the Atlantic is approximately $0.75 \mathrm{PW}$ and is located at $16^{\circ} \mathrm{N}$. The maximum overturning rate in the basin at this latitude is about $14 \mathrm{~Sv}$ (Fig. 5). In the Pacific Ocean, heat is transported poleward in both hemispheres, in general agreement with observations (Macdonald and Wunsch 1996). Notice that the model Pacific simulates the effects of Indian and Pacific Oceans combined. The modeled Atlantic heat transport, on the other hand, is southward south of $10^{\circ} \mathrm{S}$, in conflict with observations (e.g., Macdonald and Wunsch 1996).

Values from Macdonald and Wunsch (1996) are also shown in Fig. 6 to demonstrate that the model underestimates meridional heat transport. There are a number of possible causes for this, including surface boundary conditions, coarse resolution, the parameterization of 

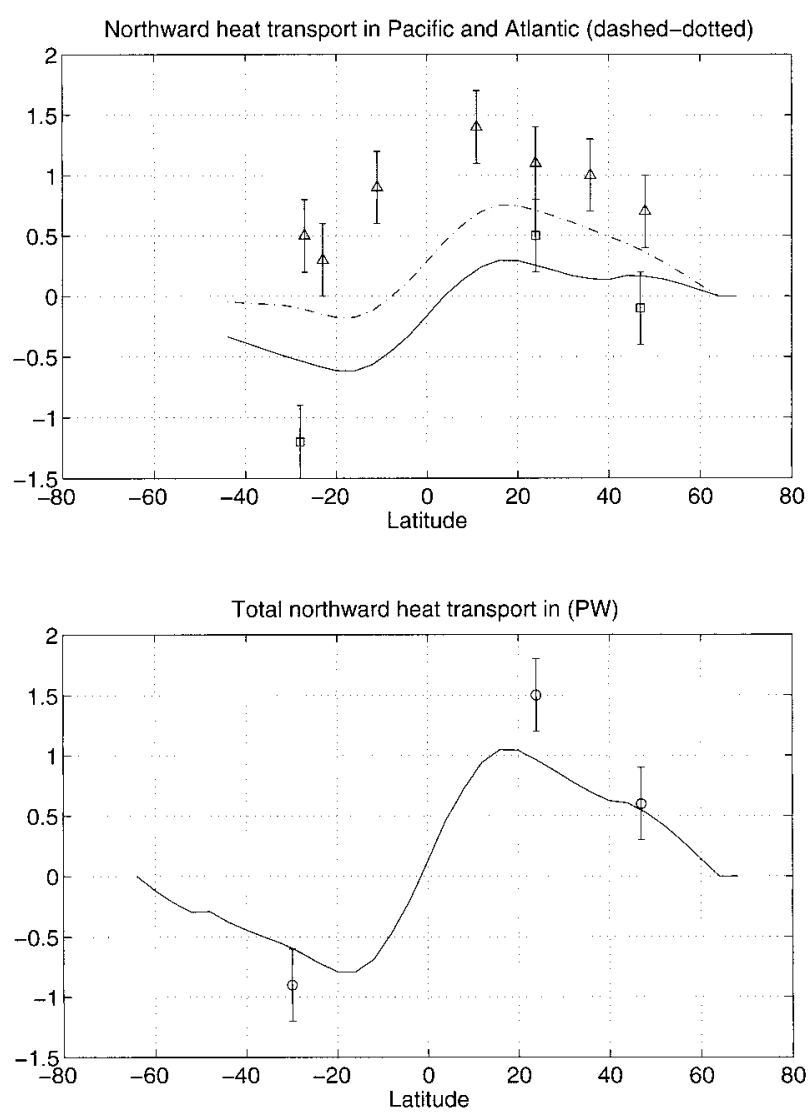

FIG. 6. Northward heat transport in experiment ZONAV (petawatts). (a) Heat transports in Pacific basin (solid line) and Atlantic basin (dashed-dotted line); the region of ACC is not included. Macdonald and Wunsch values are shown by squares for Indo-Pacific and triangles for Atlantic with corresponding errorbars. (b) Total heat transport in both basins. Macdonald and Wunsch values are shown by circles with errorbars.

unresolved mesoscale eddy fields, and highly idealized basin geometry and topography. The following experiments are devoted to the investigation of the relative effects of some of these factors. We have the dual goal of understanding what controls heat transport strength and of determining how realistic transport strengths can be obtained in an idealized model, which is a prerequisite for coupled (ocean-atmosphere) process studies (Marotzke and Stone 1995; Weaver and Hughes 1996).

\section{b. Finite zonal mixing: Experiments ATMIXB and ATMIX}

The change from the setup of experiment ZONAV is in the parameterization of the zonal mixing of surface temperature. Instead of zonal averaging of SST, we introduce a relaxation of surface temperature $T_{s}$ to its zonal mean $\bar{T}_{s}$. The following term is then used as part of the surface flux $F_{H}$ in Eq. (2):

$$
M_{\mathrm{zon}}=\rho C_{p} d_{1} \frac{\left(\overline{T_{s}}-T_{s}\right)}{\tau},
$$

where $\tau$ is a relaxation timescale, equal to 60 days, and $d_{1}$ is the depth of the upper layer, approximately $50 \mathrm{~m}$. The zonal mean of the above term is zero at any given latitude and therefore does not directly change the total meridional heat transport in the equilibrium.

We first define $\bar{T}_{s}$ as a basin mean and therefore neglect interbasin mixing (experiment ATMIXB). The important change from experiment ZONAV brought in by the term $M_{\text {zon }}$ is the existence of zonal SST gradients. The resulting increased zonal gradients of surface density enhance the THC (Fig. 7). The rate of NADW formation becomes almost $30 \mathrm{~Sv}$, increasing by almost $25 \%$. In contrast, the overturning rate at $16^{\circ} \mathrm{N}$, where the heat transport peaks in both basins (Fig. 7), is unchanged. The location of the maximum overturning moves deeper by one level. The deepening of the overturning cell comes together with an increase in the temperature of the deep ocean. For example, the bulk of deep water is now warmer than $5^{\circ}$ in the Atlantic. The reason for the latter change is strong advection of the SST by the model Gulf Stream, which leads to the increase in the surface temperature in the North Atlantic, where deep convection takes place. In fact, SST north of $40^{\circ} \mathrm{N}$ in ATMIXB is more than $2^{\circ}$ warmer than in ZONAV (Fig. 8). The increase in the deep Pacific is less pronounced and is most likely caused by warmer deep waters entering from the ACC.

The increase in the deep ocean temperature results in a smaller vertical temperature contrast, which counteracts the strengthening of the THC, in its effect on the heat transport. As we can see in Figs. 9 and 10, which show total heat transports for each basin from all experiments, the changes in heat transport from ZONAV to ATMIXB are small in either basin. We now look at the heat transport components, which are compared between three experiments in Fig. 11. The overturning component changes insignificantly in the Pacific and is unchanged in the Atlantic. In contrast, a baroclinic gyre component significantly increases at midlatitudes in both basins due to a larger zonal temperature contrast in horizontal gyres (Wang et al. 1995). In other words, the surface waters transported poleward by the western boundary currents in the North Atlantic are now allowed to be warmer than the interior water flowing southward at the same latitude. The two remaining components, barotropic gyre and diffusive components, are practically unchanged and remain small in amplitude.

Next, we introduce zonal mixing of temperature between the two basins by restoring the temperature to its global zonal mean instead of a basin mean (experiment ATMIX). The most immediate effect is in the SST in the northern parts of both basins. Surface waters in the North Pacific are relatively stagnant (Fig. 12) and therefore colder than the North Atlantic waters, which come from the south. The temperature mixing between two basins therefore cools the surface of the North Atlantic, which can be seen in Fig. 8 .

The change in the surface density strongly affects the 
a) Meridional overturning in the Pacific (Sv)

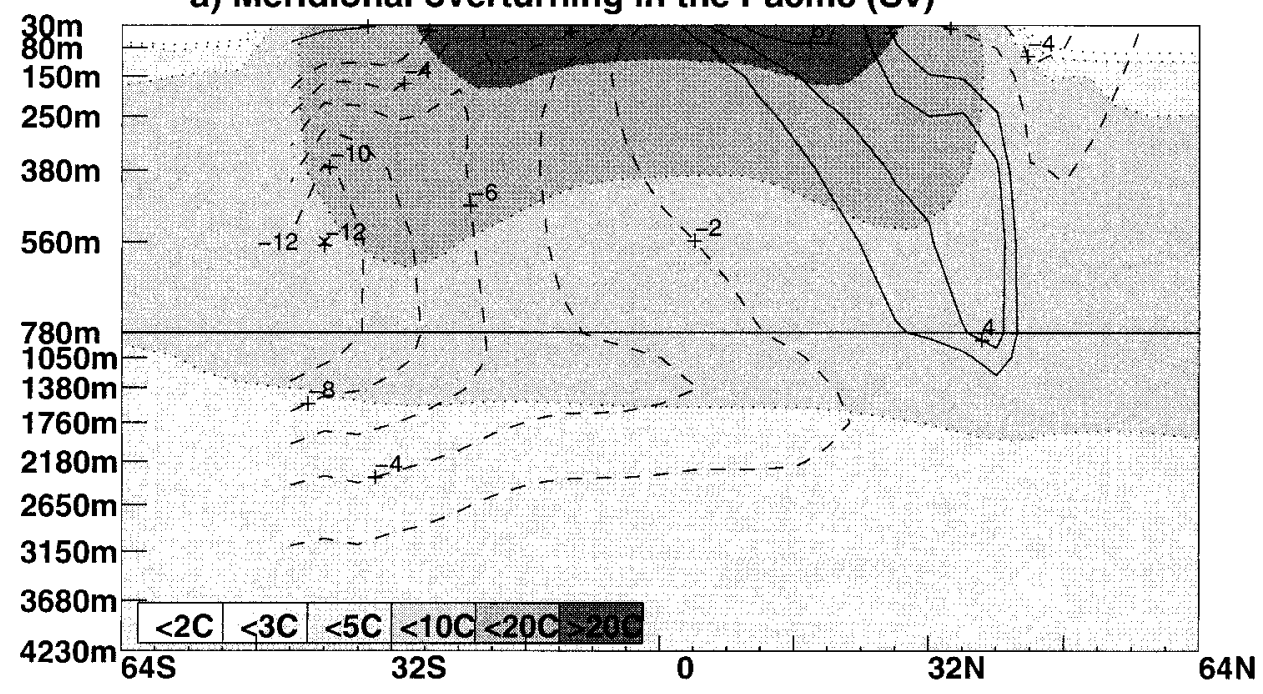

b) Meridional overturning in the Atlantic (Sv)

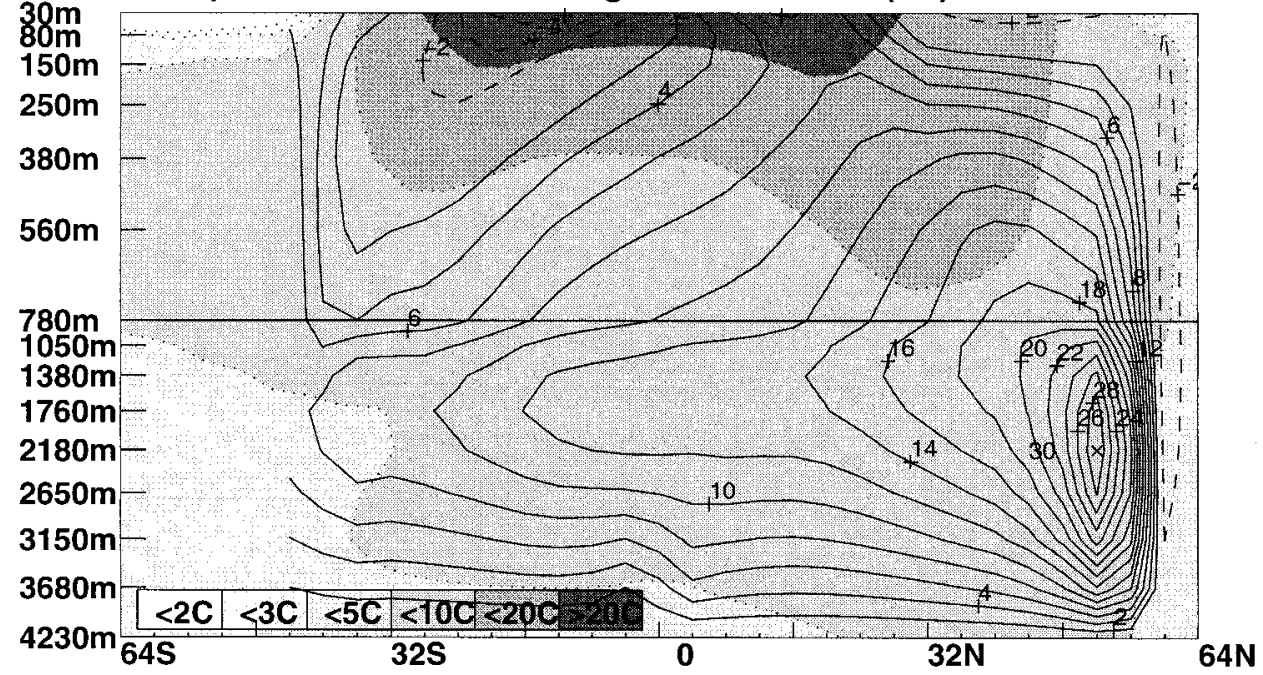

FIG. 7. Meridional overturning in experiment ATMIXB: finite basin zonal mixing. (a) Pacific and (b) Atlantic. The contours with shading repesent zonally averaged temperatures; corresponding intervals are given in the plot.

THC. As illustrated in Fig. 12, the maximum overturning rate increases to almost $34 \mathrm{~Sv}$, and its location moves northward to $60^{\circ} \mathrm{N}$. The weak upwelling at the northern boundary in Figs. 5b and 7b disappears. A larger change occurs in the overturning rate at $16^{\circ} \mathrm{N}$, which increases from 14 to almost $19 \mathrm{~Sv}$. The rate of upwelling in the Pacific basin is now reduced from 12 to $8 \mathrm{~Sv}$, so the global deep-water formation rate remains almost constant (see Tziperman 1997; WSM).

The vertical temperature contrast increases significantly at low latitudes (Fig. 12), a result of colder waters sinking in the North Atlantic. The ocean below $1500 \mathrm{~m}$ is now significantly colder than in experiment ATMIXB (compare Figs. 7 and 12). The heat transport reflects the changes in the temperature structure and mass circulation. The overturning component of the heat transport increases dramatically at low latitudes in the Atlantic basin (Fig. 11b), where the heat transport peaks at almost $1.3 \mathrm{PW}$ at $16^{\circ} \mathrm{N}$. In addition, the total heat transport is now northward everywhere in the Atlantic, in contrast to southward heat transport in the South Atlantic in experiments ZONAV and ATMIXB (Fig. 10).

The southward heat transport by vertical overturning north of $26^{\circ} \mathrm{S}$ in the Pacific is larger than in experiments 


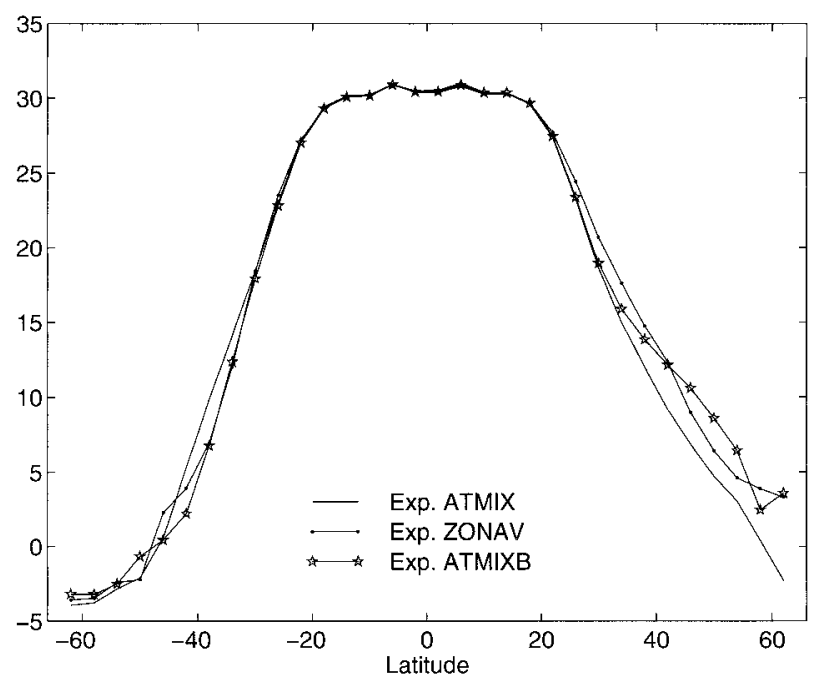

FIG. 8. Zonally averaged SST in the Atlantic basin in experiment ATMIX (solid line), experiment ATMIXB (pentagrams), and ZONAV (dots).

ZONAV and ATMIXB (Fig. 11a). The increase in the vertical temperature contrast in the basin apparently overcomes the effect of weakened circulation in the Southern Pacific. At $16^{\circ} \mathrm{N}$, the Ekman cell is now shallower; which results in weaker northward heat transport at that latitude (Figs. 12a, 5a, and 11).

The baroclinic gyre components also increase at midlatitudes where the strong western boundary currents are located (Figs. 11c and 11d). The most significant change occurs in the North Atlantic where the gyre heat transport becomes a significant contributor to the total. Interbasin mixing mainly cools the interior of the gyre where surface waters flow sluggishly, whereas the water particles near the western boundary are moving too fast to be significantly affected. The result is further enhancement of the zonal temperature contrast and increase in the gyre components of the heat transport.

\section{c. Fast western boundary currents: Experiment FAST}

The western boundary currents advect warm waters poleward, which has two main dynamical effects. As shown in the preceding section, increases in the zonal gradients of SST influence the strength of the THC. More importantly, larger zonal temperature contrasts directly enhance heat transport by the horizontal gyres (Wang et al. 1995; Fanning and Weaver 1997).

The surface speeds of western boundary currents are underestimated in coarse-resolution models; in our case the maximum surface meridional velocity in the North Atlantic is $25 \mathrm{~cm} \mathrm{~s}^{-1}$, which is well below the observed values of 100 to $200 \mathrm{~cm} \mathrm{~s}^{-1}$ (e.g., Johns et al. 1995) and causes too-weak heat transport (Wunsch 1996; Fanning and Weaver 1997).

To alleviate this problem somewhat, we decrease the value of the horizontal viscosity from $2.5 \times 10^{5} \mathrm{~m}^{2} \mathrm{~s}^{-1}$

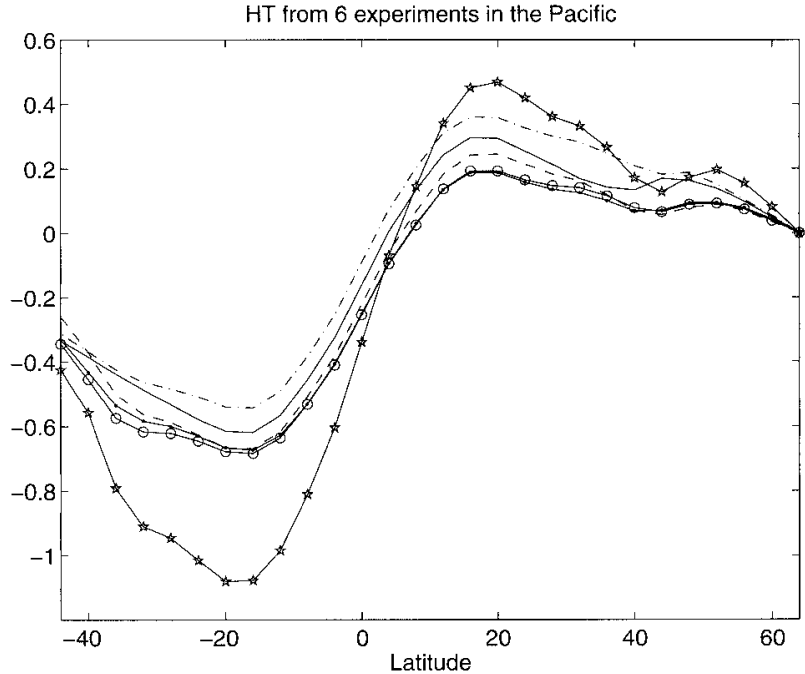

FIG. 9. Comparison of total heat transport in the Pacific from six experiments. Solid lines are ZONAV, dashed-dotted are ATMIXB, solid-dotted are ATMIX, circles are FAST, dashed are GM, and pentagrams are WIDE.

to $0.5 \times 10^{5} \mathrm{~m}^{2} \mathrm{~s}^{-1}$, making the characteristic width of the viscous boundary currents $240 \mathrm{~km}$ (Munk 1950). The zonal resolution now varies from $1.25^{\circ}$ at the boundaries to $3.75^{\circ}$ in the interior of each basin. Although the new resolution is still too coarse to properly resolve frontal features in the western boundary regions, it is sufficient for illustrating the effect of increased resolution.

The meridional overturning (Fig. 13) changes very little compared to experiment ATMIX. The maximum rate of sinking decreases from $34 \mathrm{~Sv}$ in experiment ATMIX to $31 \mathrm{~Sv}$ in the present case, and the location of

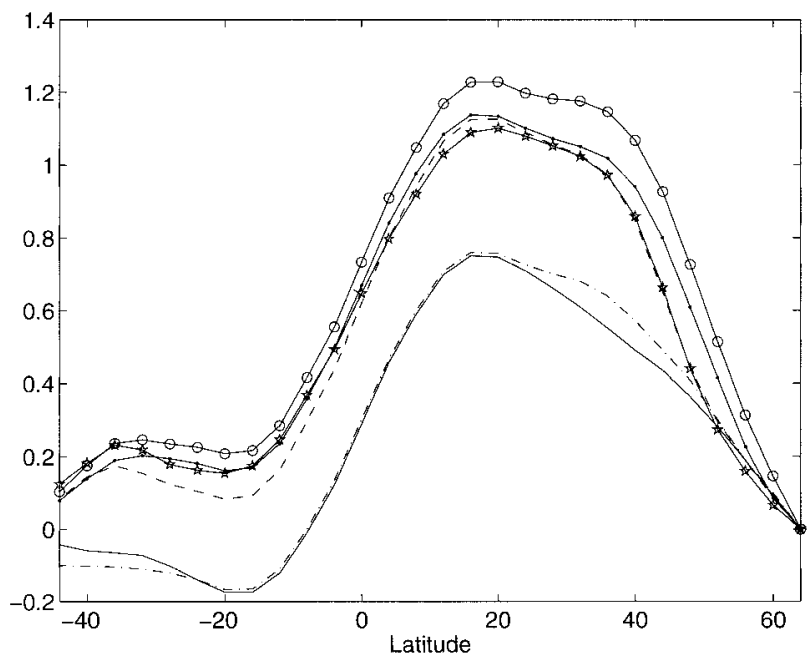

FIG. 10. Comparison of total heat transport in the Atlantic from six experiments. Solid lines are ZONAV, dashed-dotted are ATMIXB, solid-dotted are ATMIX, circles are FAST, dashed are GM, and pentagrams are WIDE. 
a) Overturning comp. in the Pacific

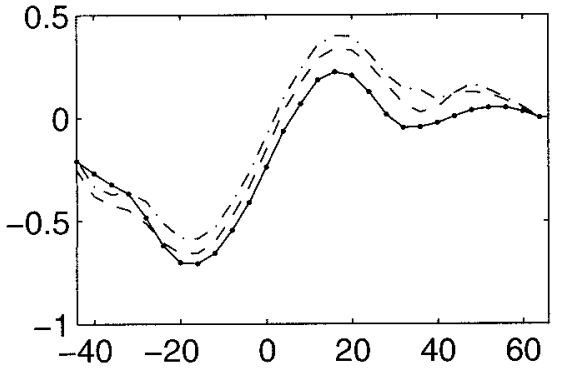

c) Baroclinic gyre comp. in the Pacific

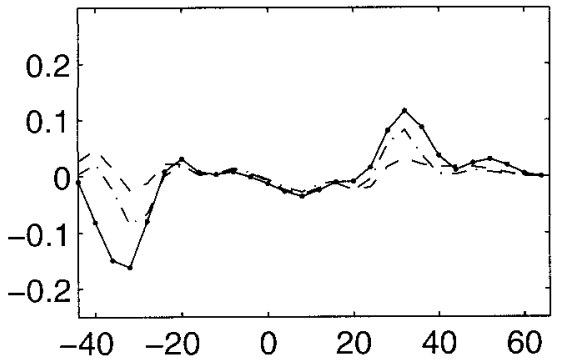

e) Barotropic gyre comp. in the Pacific

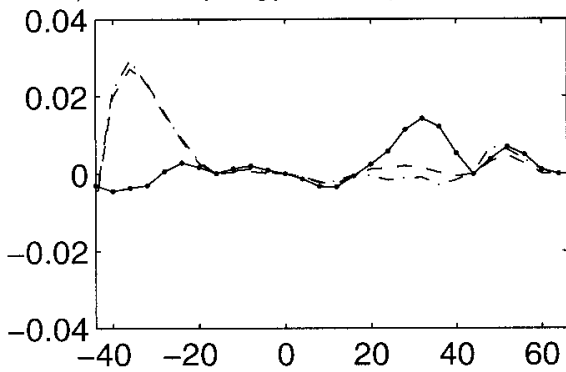

g) Diffusive comp. in the Pacific

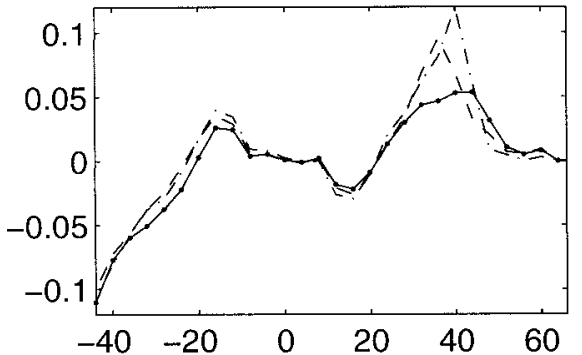

b) Overturning comp. in the Atlantic

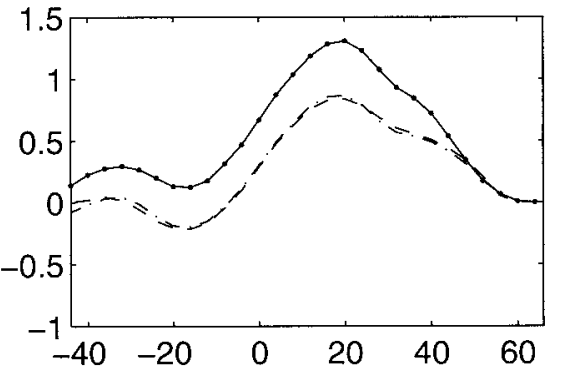

d) Baroclinic gyre comp. in the Atlantic

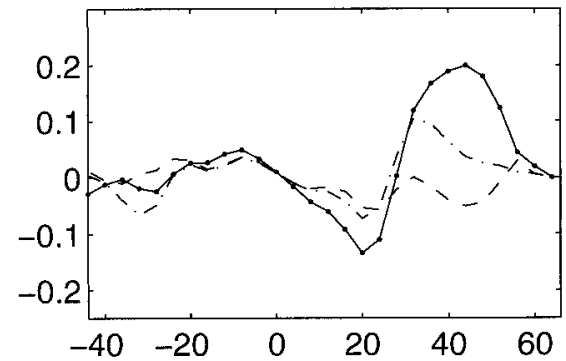

f) Barotropic gyre comp. in the Atlantic

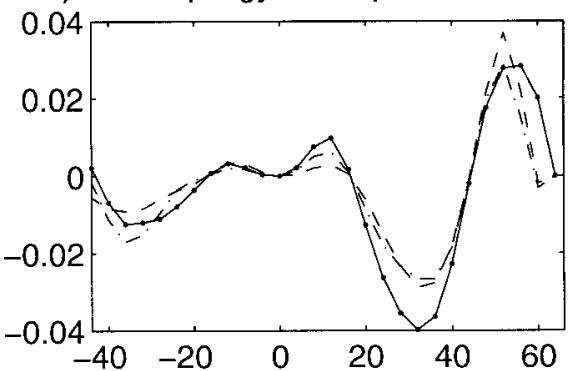

h) Diffusive comp. in the Atlantic

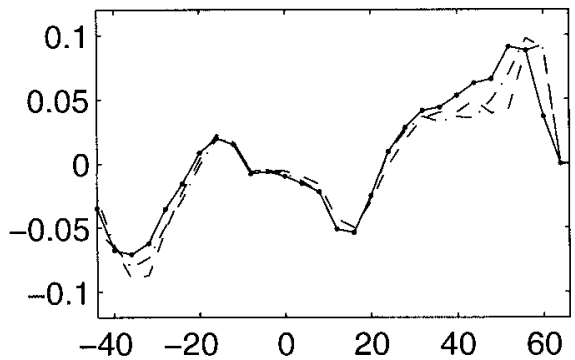

FIG. 11. Comparison of heat transport components in experiment ZONAV (dashed lines), experiment ATMIXB (dashed-dotted lines) and experiment ATMIX (solid-dotted lines). Left panels show the Pacific; right panels show the Atlantic. First row: overturning components; second row: baroclinic gyre components: third row: barotropic gyre components; and fourth row: diffusive components.

the maximum mass transport moves southward from $60^{\circ}$ to $52^{\circ} \mathrm{N}$. Net upwelling in the North Atlantic is also weaker, leading to slightly larger cross-equatorial flow.

The increased speed of the western boundary currents and associated sharpening of the frontal features tend to increase horizontal diapycnal mixing in the boundary current regions (Böning et al. 1995). According to MK, increased mixing in locations outside the North Atlantic (away from the dominant deepwater source) leads to an increase in the cross-equatorial mass transport, while increased mixing in the North Atlantic causes a reduction. In our experiment, the mixing is increased across all boundary current fronts. However, upwelling occurs in the larger portion of the model domain, so one would expect larger cross-equatorial transport with generally sharper boundary currents.

The increased resolution and faster boundary currents lead to an increase in total heat transport, particularly in the North Atlantic (Fig. 10). The overturning components of the heat transport increase in the present experiment 

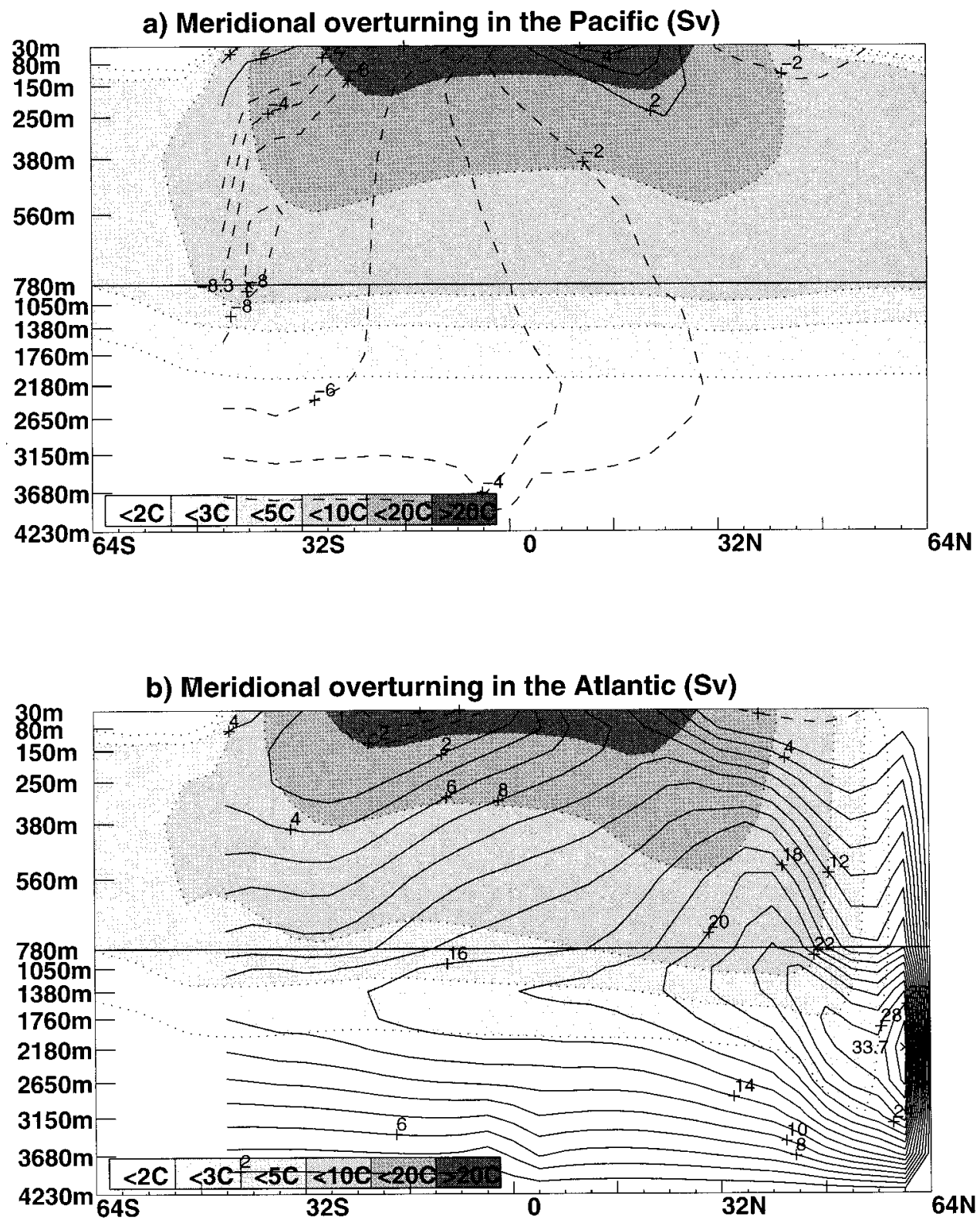

FIG. 12. Meridional overturning in experiment ATMIX: Interbasin zonal mixing. (a) Pacific and (b) Atlantic. The contours with shading represent zonally averaged temperatures; corresponding intervals are given in the plot.

by less than $0.1 \mathrm{PW}$ (see Fig. 14), which is a small increase in relative terms. In contrast, the changes in the gyre components are noticeable, especially in the Atlantic. The baroclinic component increases by more than 0.1 PW $(30 \%)$ in the region of the western boundary current in the North Atlantic (approximately $40^{\circ} \mathrm{N}$ ). The barotropic component nearly doubles in amplitude in the North Atlantic because of the increased zonal SST gradient, but remains small compare to other advective components. The diffusive component almost doubles its amplitude at high latitudes in the North Atlantic, which indicates larger vertically integrated meridional temper- ature gradients. The changes are somewhat weaker in the Pacific. The observed increase is consistent with findings by Fanning and Weaver (1997) in terms of absolute value of the changes. Their large relative increase is attributed to the very small magnitude of total heat transport in their single-hemisphere model.

\section{d. Gent-McWilliams parameterization: Experiment GM}

Up to this point, we have parameterized the eddy transports by horizontal and vertical diffusion with con- 


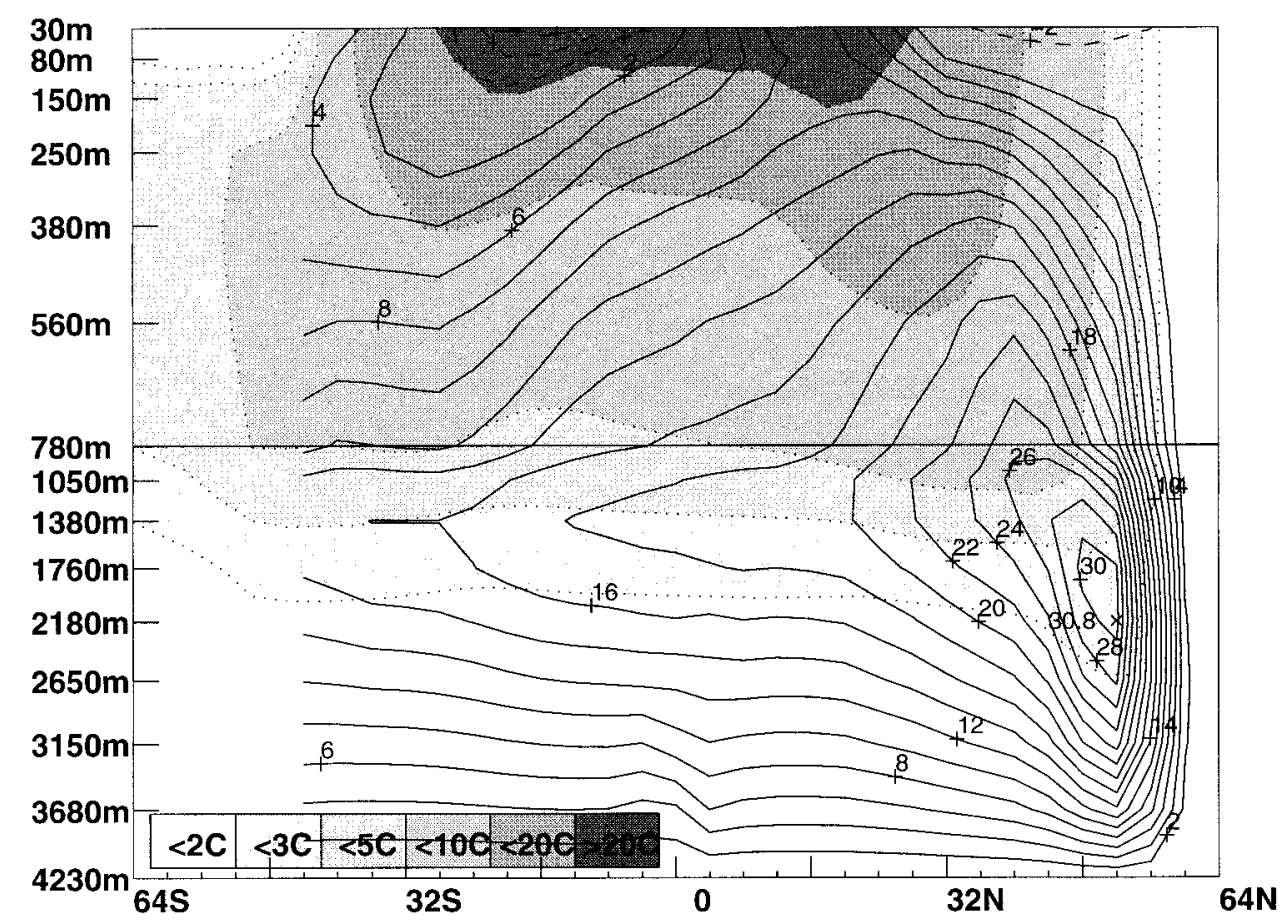

FIG. 13. Meridional overturning streamfunction (Sv) in experiment FAST in the Atlantic basin. The contours with shading represent zonally averaged temperatures; corresponding intervals are given in the plot.

stant coefficients. We now switch to a combination of isopycnal mixing and "eddy-induced" transports (GM scheme: see Gent and McWilliams 1990; Danabasoglu et al. 1994). The eddy transports are computed from the assumption of downgradient diffusion of the thickness between neighboring isopycnal surfaces with constant diffusion coefficients.

The most conspicuous effect of the GM scheme is a decrease in the overturning strength in the Atlantic (Fig. 15), consistent with Weaver and Eby (1997), Duffy et al. (1997), and Jiang et al. (1999, manuscript submitted to J. Phys. Oceanogr.), and most likely related to the overall reduction in the diapycnal mixing in the model (Gerdes et al. 1991). Indeed, the replacement of the horizontal tracer diffusion by the isopycnal mixing eliminates an additional cross-isopycnal mixing arising from sloping isopycnal surfaces.

The upper panel of Fig. 15 shows the Eulerian mean circulation without adding the eddy-induced velocities in the Atlantic. We see a reduction in the sinking rate from $30 \mathrm{~Sv}$ in experiment FAST to $19 \mathrm{~Sv}$ in the present case. The location of the maximum overturning now moves south to $44^{\circ} \mathrm{N}$. The overturning rate at the point of maximum heat transport (Fig. 16) is about $16 \mathrm{~Sv}$, also down from $19 \mathrm{~Sv}$ in the preceding experiment. The amount of water reaching the ACC region is also reduced by almost $4 \mathrm{~Sv}$. The reduction in the overturning at low latitudes is also consistent with the decreased diapycnal mixing in the regions with upwelling (see preceding section and $\mathrm{MK}$ ).

The weakening of THC in principle leads to a decrease in the heat transport in the model. However, there are two additional effects of the GM parameterization that both act to increase the heat transport.

The first effect is the decrease in temperature of the deep ocean (cf. Figs. 15a and 13). There are several reasons for the change. Reduced circulation implies that water particles spend more time at the surface at high latitudes and are therefore cooled more strongly by the cold atmosphere above. The result is lower temperatures at high latitudes in the North Atlantic, which are linked to the deep ocean by convection. The decrease in diapycnal mixing also contributes to stronger stratification and a generally sharper thermocline (Danabasoglu and McWilliams 1995). Furthermore, the vertical mixing efficiency (Gerdes et al. 1991) is not spatially uniform if isopycnal mixing is employed. Instead, the vertical mixing is increased in the regions of steep isopycnal surfaces; therefore, vertical mixing at high latitudes is increased, which cools the deep ocean further. In other words, the isopycnal mixing connects the deep ocean interior to the cold surface waters at high latitudes where isopycnal surfaces tend to outcrop (Duffy et al. 1997). In addition, the convective activity in the Southern Ocean is dramatically reduced by the introduction of the GM scheme, in good agreement with the results by 
a) Overturning comp. in the Pacific

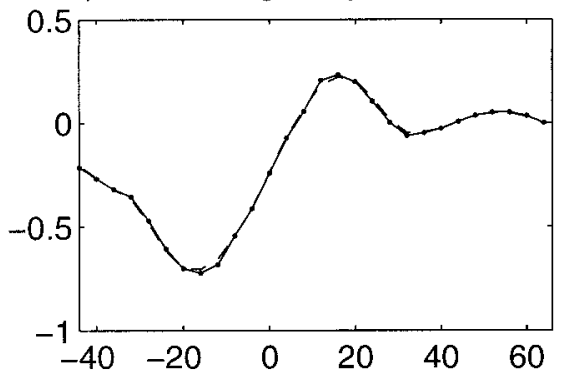

c) Baroclinic gyre comp. in the Pacific

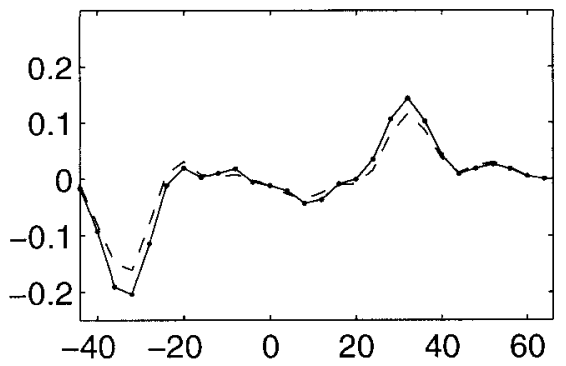

e) Barotropic gyre comp. in the Pacific

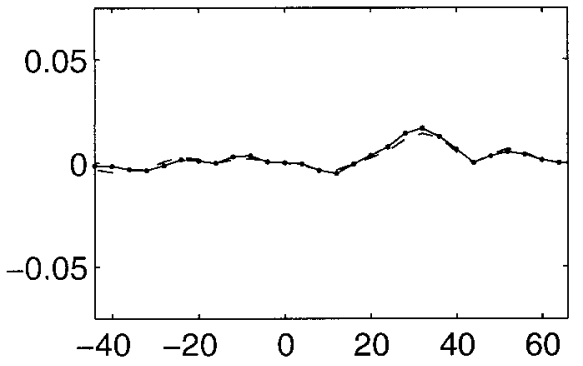

g) Diffusive comp. in the Pacific

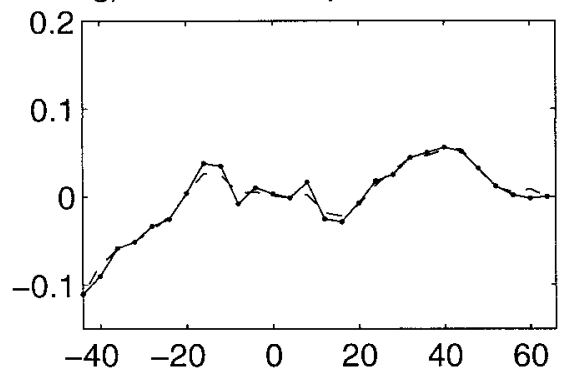

b) Overturning comp. in the Atlantic

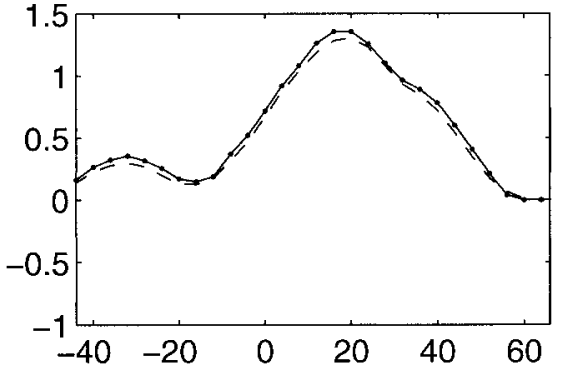

d) Baroclinic gyre comp. in the Atlantic

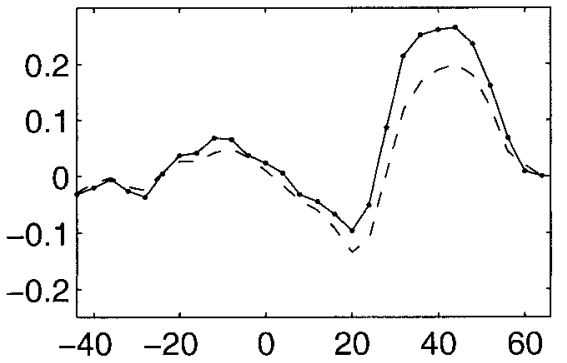

f) Barotropic gyre comp. in the Atlantic

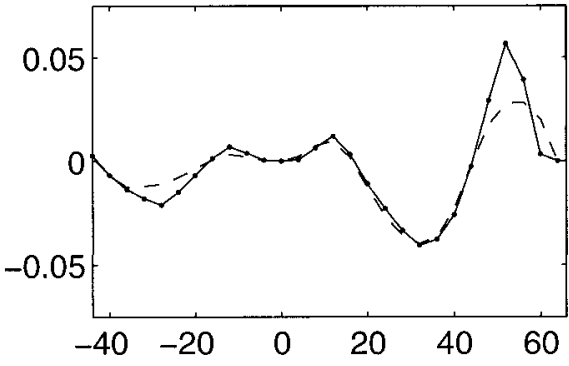

h) Diffusive comp. in the Atlantic

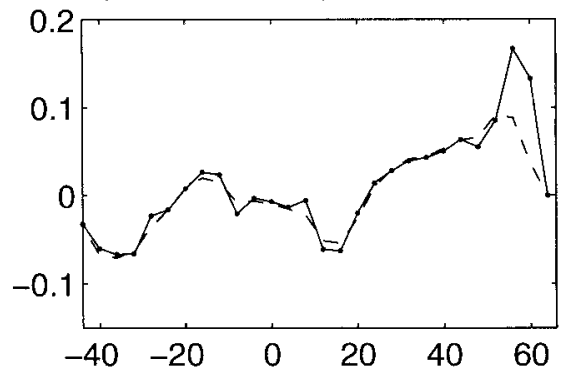

FIG. 14. Comparison of heat transport components in experiment ATMIX (dashed lines) and experiment FAST (solid-dotted lines). Left panels show the Pacific, right panels show the Atlantic. First row: Overturning components; second row: Baroclinic gyre components; third row: Barotropic gyre components; fourth row: Diffusive components.

Danabasoglu and McWilliams (1995). Convection at the northern edge of the ACC in experiment FAST establishes a direct link between warmer surface waters and deeper layers. Elimination of the convection therefore contributes to cooling the deep ocean.

Another effect of the GM scheme is the existence of eddy-induced bolus transport velocities (Gent and McWilliams 1990). They are added to the mean field in Fig. 15b, which increases the transport of temperature and salinity. The change is, however, rather small; in fact, the increase in mass transport is noticeable only in the vicinity of the northern boundary where intensive convection takes place and the isopycnals are steep.

As a result of the several competing dynamical effects described above, heat transport decreases compared to experiment FAST. Heat transport components are shown in Fig. 16; overturning and gyre components now include transports by eddy-induced velocities. One can 

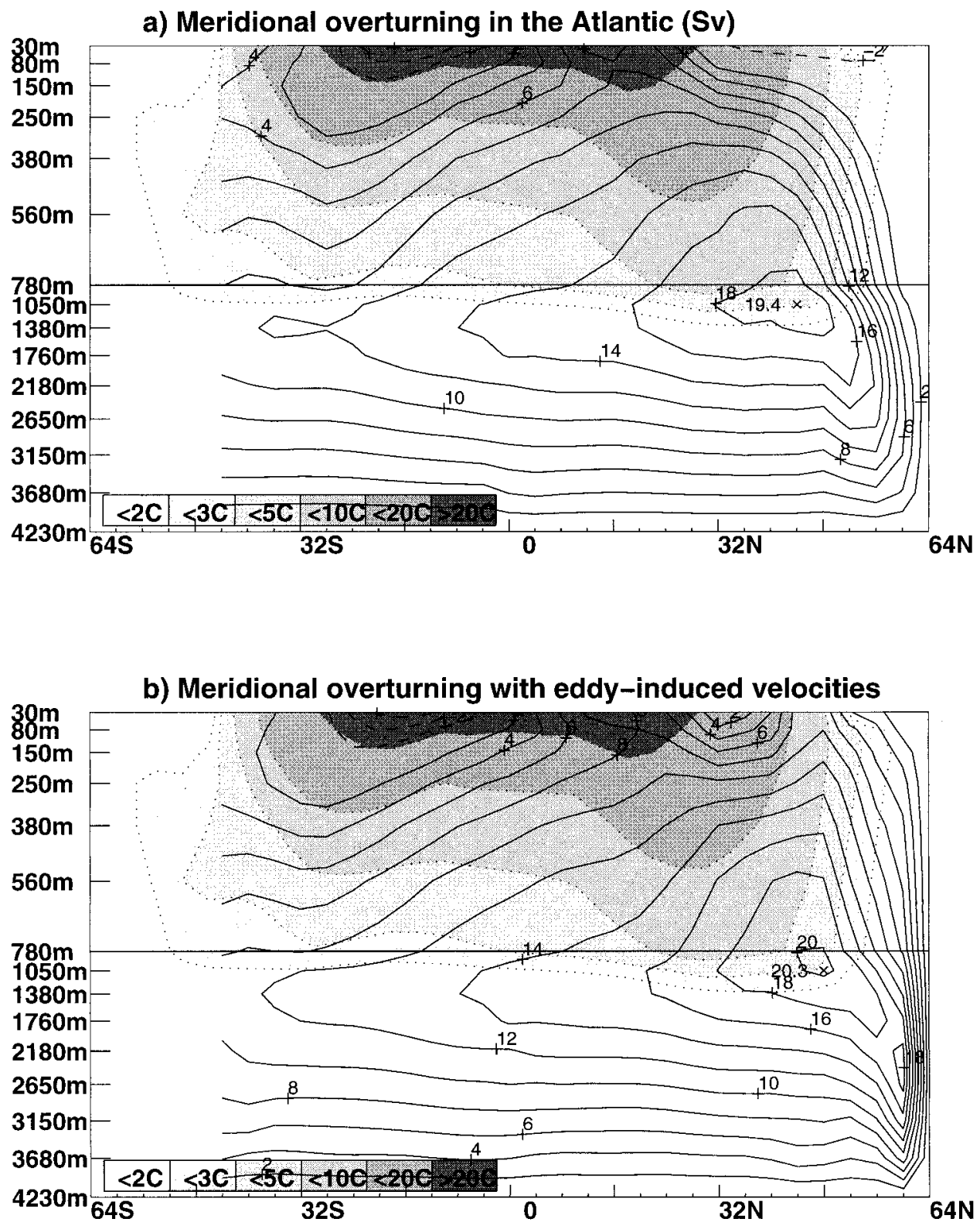

FIG. 15. Meridional overturning streamfunction (Sv) in experiment GM, the Atlantic basin only. (a) Mean circulation. In (b) eddy-induced transport velocities are added; see text for details. The contours with shading represent zonally averaged temperatures; corresponding intervals are given in the plot.

see small decreases in the various components in the Atlantic basin, generally 0.1 to $0.2 \mathrm{PW}$. The reduction in the overturning component is caused by the reduction of the mass transport of the THC.

A second change is a much larger decrease in the baroclinic gyre component in the midlatitudes of the North Atlantic. There are several reasons for the change. First, weaker zonal-mean circulation in experiment GM leads to a weaker gyre and slower surface boundary currents. As a result of weaker advection of temperature in boundary layers, zonal SST gradients are also reduced near the surface, as our analysis shows (see also section 4b). Since horizontal heat flux by gyres is concentrated in upper layers, both factors lead to its reduction in experiment GM. In addition, eddy-induced bolus velocities (included in upper six panels of Fig. 16) appear to further contribute to the weakening of the component. The changes in the Pacific are less pronounced.

The diffusive component, which now is the horizontal component of isopycnal diffusive flux, is considerably smaller at mid and high latitudes in both basins. Reduced horizontal gradients of temperature (not shown here) partly account for the change. In addition, isopycnals are not horizontal, which results in a smaller 
horizontal projection of the diffusive fluxes. The overall result is a considerable reduction in the total northward heat transport in the Atlantic basin (Fig. 10).

\section{e. Gent-McWilliams parameterization with wide Pacific: Experiment WIDE}

The last experiment is focused on the role of a basin's width. According to Wang et al. (1995), gyre heat transport should increase with basin width or, more precisely, with zonally integrated Sverdrup transport. The dependance on basin width should be between linear and quadratic, depending on the strength of atmospheric coupling. In experiment WIDE, we make the Pacific 120 degrees wide, twice as wide as in all other experiments. The changes in the Atlantic basin, whose width remain unchanged, are of particular interest. In addition to the anticipated change in the circulation in the basin induced by the larger area of mixing in the Pacific, the surface heat flux also changes. In the formulation of the surface boundary conditions, we explicitly assume that all heat leaving the atmosphere at its bottom enters the ocean. The oceanic surface heat flux is multiplied by the ratio of $360^{\circ}$ to the total width of the oceans in degrees [the factor $1 / \epsilon$ in Eq. (2)]. When the width of the Pacific is doubled and the size of the Atlantic is unchanged, the factor is now 2 instead of 3 and the fixed part of the surface heat flux decreases in the Atlantic. It remains to be seen if Atlantic SST can adjust to compensate this change.

The model is integrated to equilibrium in a way similar to all preceding experiments, with one interesting difference: The conveyor belt equilibrium is obtained without applying anomalous freshwater flux (section 2). In experiment ZONAV with symmetric geometry, these anomalous fluxes are needed to drive the model from a "northern sinking" state to a "conveyor belt" equilibrium. In WIDE, deep sinking in the North Pacific is inhibited, consistent with Hughes and Weaver (1994) but for reasons that are not understood.

As a consequence of the widening of the basin, the mass transport in the Pacific increases to $11 \mathrm{~Sv}$ at its maximum (Fig. 17) right at the boundary with the ACC. The changes in THC strength in the Atlantic basin are rather small (cf. Figs. 15b and 17b). In particular, the mass overturning at the latitude of maximum heat transport in the basin $\left(16^{\circ} \mathrm{N}\right)$ increases by about $2 \mathrm{~Sv}$ from $15 \mathrm{~Sv}$ in experiment GM to $17 \mathrm{~Sv}$. The maximum overturning rate in the North Atlantic increases by the same $2 \mathrm{~Sv}$ to become $22.7 \mathrm{~Sv}$; the amount of mass transport that is short-circuited in the Northern Hemisphere is unchanged. However, the amount of deep water leaving the Atlantic increases from 10 to $14 \mathrm{~Sv}$. We also observe that the deep ocean is warmer than in experiment GM.

Klinger and Marotzke (KM) presented a scaling analysis showing that, in a single basin, thermocline depth scales with the one-third power of basin width and overturning strength scales with the two-thirds power. Ba- sically, this dependence arises because in a wider basin with the same meridional density contrast, the upwelling mass transport is distributed over a wider area. Consequently, the upwelling velocity is weaker, which deepens the thermocline and leads to an increase in overturning. Qualitatively, these arguments are confirmed in our experiments, although the explanation is not quantitatively satisfactory. Possible complicating factors are the existence of two major deep-water sources in our model (North Atlantic and Southern Ocean), rather than one source as in the asymmetric solutions of KM and the general concentration of upwelling near the boundaries (Marotzke 1997), which might reduce the effect of changes in width.

Widening the Pacific has a negligible influence on Atlantic heat transport, but nearly doubles the poleward heat transport in the Pacific, at all latitudes (Fig. 9). The heat transport components are shown in Fig. 18; the overturning component in the Pacific doubles as a result of the doubled mass transport. The gyre components also increase because of the increased speed and total mass transport of the western boundary currents in the basin. Larger Sverdrup transport is compensated in the model by the transport in the western boundary layers, whose width is unchanged, hence its velocity and temperature advection increased, which leads to increased zonal temperature differences and therefore larger gyre heat transport (Wang et al. 1995). In particular, the magnitude of the baroclinic gyre component doubles at midlatitudes, whereas the barotropic gyre component nearly triples. The diffusive component in the Pacific doubles at midlatitudes in both hemispheres.

\section{Summary and discussion}

We have presented a series of experiments to understand how several physical processes control the strength of the meridional heat transport. We compare the results of the experiments and focus on the changes in the thermohaline circulation and the heat transport caused by changes in the dynamics. We attempt here to understand the relative importance of different physical processes in affecting the meridional heat transport. The results are summarized in Figs. 9 and 10, where total heat transport in each model basin is reported for six experiments.

1) The form of zonal mixing of SST by the atmosphere has a large effect on the circulation in the Atlantic basin and its meridional heat transport. We have parameterized zonal mixing by very simple means. In one extreme, "infinitely efficient" mixing within each model basin completely homogenizes SST zonally (experiment ZONAV), which leads to a noticeably weaker Atlantic THC than in the case with finite zonal mixing (experiment ATMIXB). Large zonal density gradients in the latter case enhance the meridional overturning in the northern North Atlantic. In contrast, low-latitude overturning and hence the maximum heat transport are 
a) Overturning comp. in the Pacific

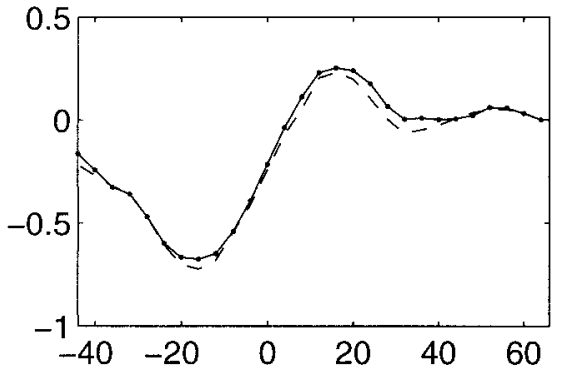

c) Baroclinic gyre comp. in the Pacific

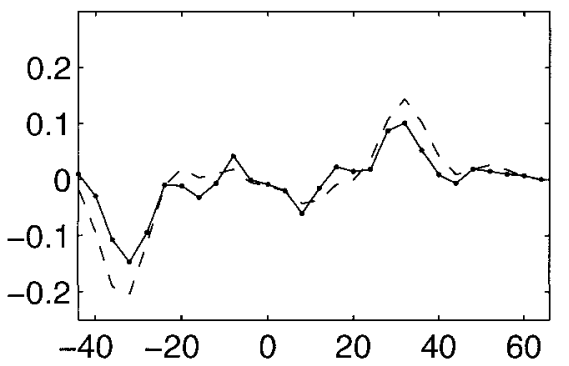

e) Barotropic gyre comp. in the Pacific

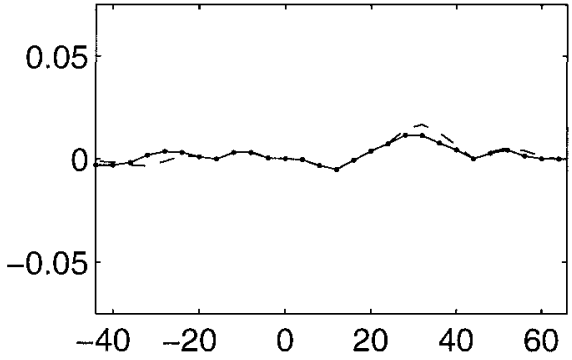

g) Diffusive comp. in the Pacific

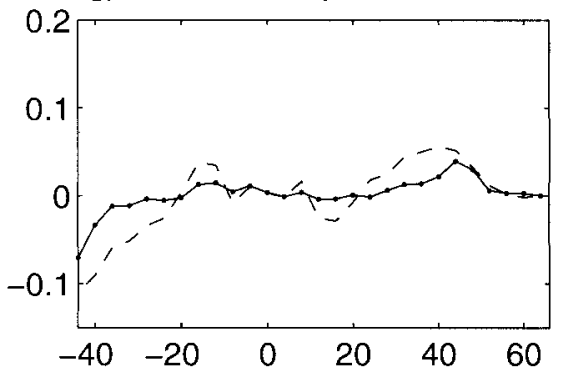

b) Overturning comp. in the Atlantic

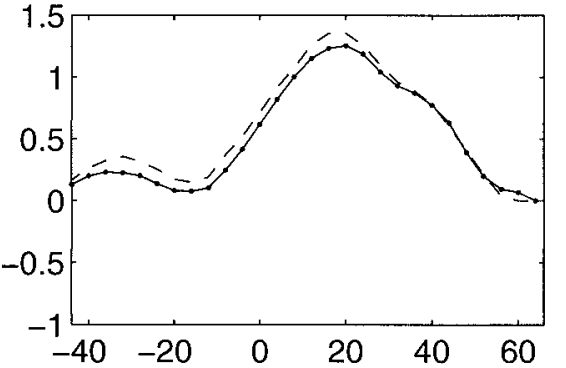

d) Baroclinic gyre comp. in the Atlantic

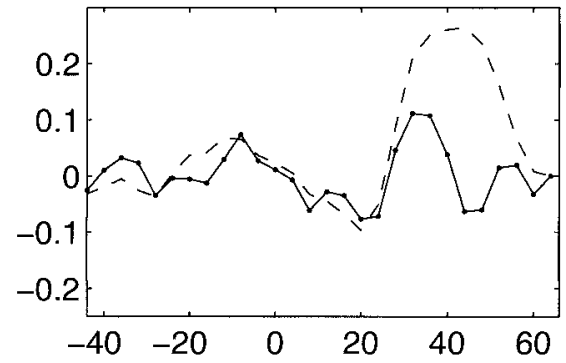

f) Barotropic gyre comp. in the Atlantic

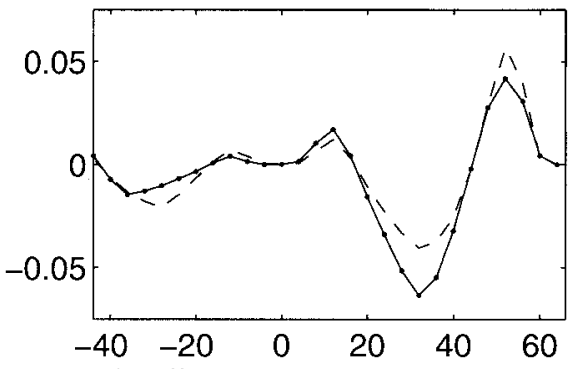

h) Diffusive comp. in the Atlantic

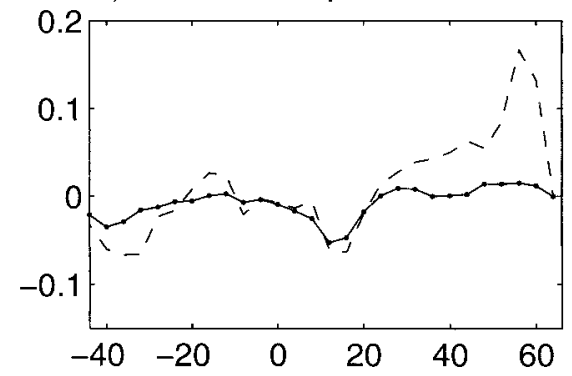

FIG. 16. Comparison of heat transport components in experiment FAST (dashed lines), experiment GM (solid-dotted lines). Left panels show the Pacific; right panels show the Atlantic. First row: overturning components; second row: baroclinic gyre components; third row: barotropic gyre components; and fourth row: diffusive components. Eddy transports are added to all advective components.

only weakly affected by the degree to which SST is zonally homogenized within each basin.

The heat transport in the Atlantic increases significantly when basin zonal mixing is replaced by interbasin mixing. The immediate effect of the process is a decrease in North Atlantic SST and resulting cooling of the deep ocean through the intense deep convection in that region. Increased vertical temperature contrast at low latitudes then leads to a larger overturning com- ponent of the heat transport, while mass circulation at mid- and low latitudes also increases.

Large zonal SST gradients in experiments ATMIXB and ATMIX lead to a larger amount of heat transported by the Atlantic horizontal gyres. The cooling of the ocean interior due to the interbasin mixing in experiment ATMIX further enhances the effect.

We have included only a crude representation of the zonal temperature mixing in our model. A more accurate 


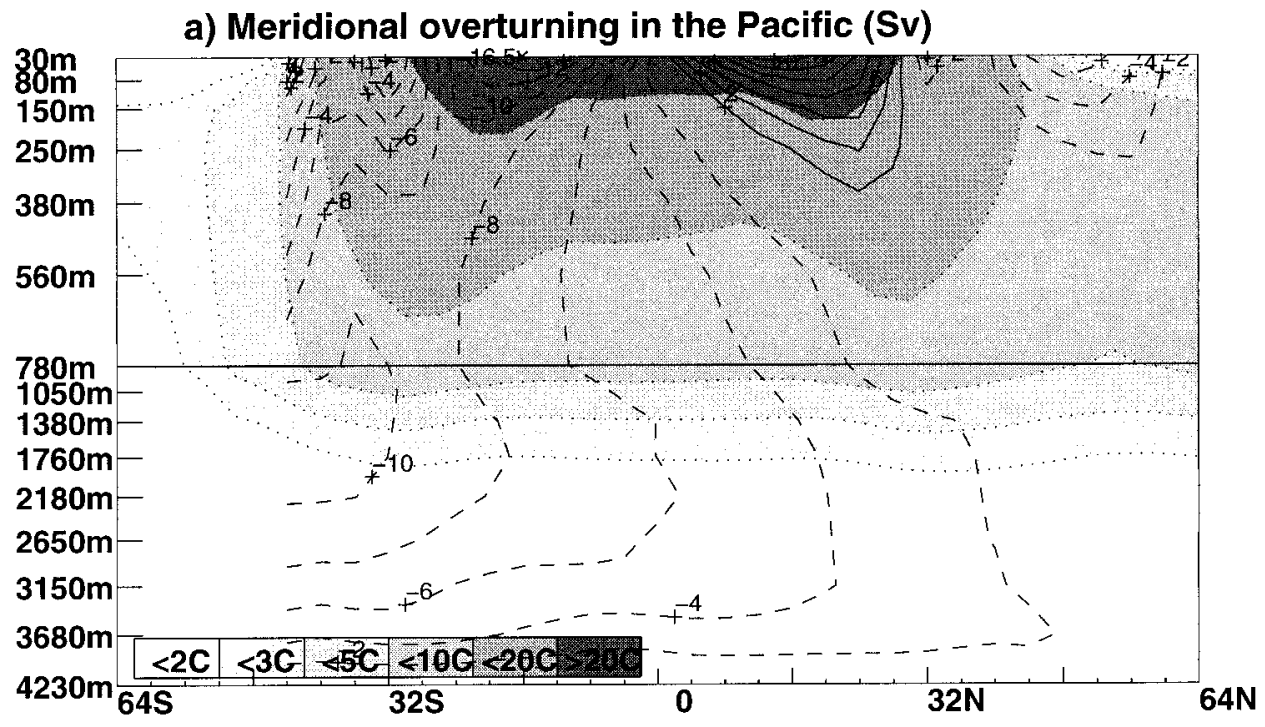

b) Meridional overturning in the Atlantic (Sv)

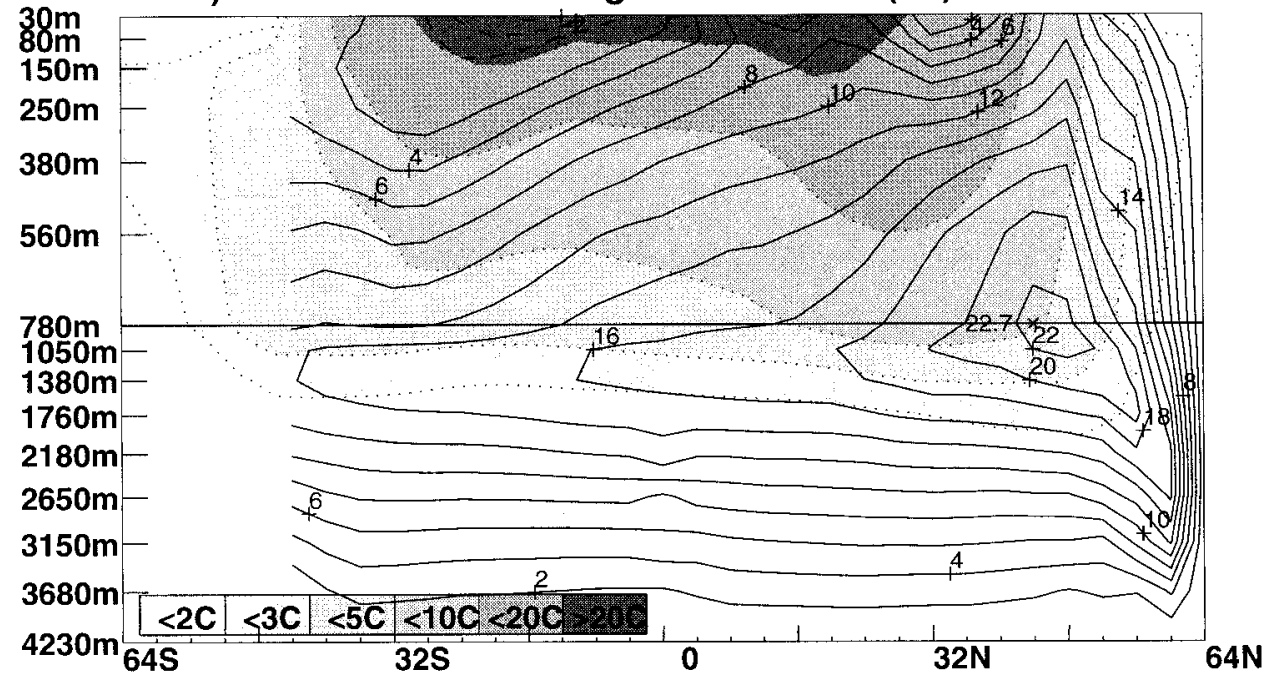

FIG. 17. Meridional overturning in experiment WIDE for (a) Pacific and (b) Atlantic. Eddy-induced transport velocities are added in the both panels. The contours with shading repesent zonally averaged temperatures; corresponding intervals are given in the plot.

parameterization of this process is needed and might change our results quantitatively. Ultimately, a fully coupled atmosphere-ocean GCM is required for a complete representation of the temperature feedbacks (Mikolajewicz et al. 1997). However, our study indicates the important role of the zonal mixing process in controlling oceanic heat transport. The results also show the different roles of intrabasin and interbasin heat exchanges. The latter clearly has the largest effect on the heat transport by exchanging heat between the relatively cold North Pacific and warm North Atlantic.

2) To study the effects of the western boundary cur- rents in the model, we contrast the results of experiment ATMIX and experiment FAST. In the latter case, the width of western boundary currents is nearly halved, leading to a substantial increase in the current speed. Stronger western boundary currents lead to a insignificant increase in the overturning components of the meridional heat transport. However, the increase in the gyre components is significant in the Atlantic, in a good quantitative agreement with the results by Fanning and Weaver (1997). The meridional resolution is unchanged in our model; an increase in only the zonal resolution of boundary currents and in their speed are apparently 
a) Overturning comp. in the Pacific

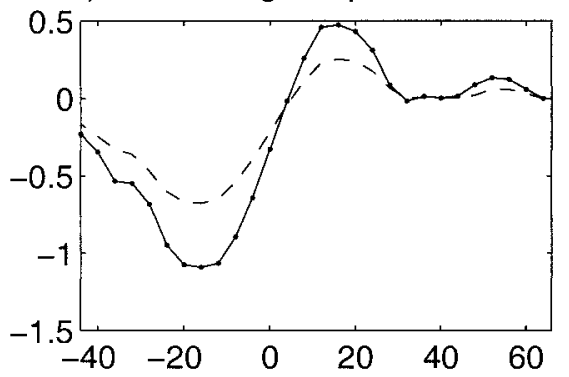

c) Baroclinic gyre comp. in the Pacific

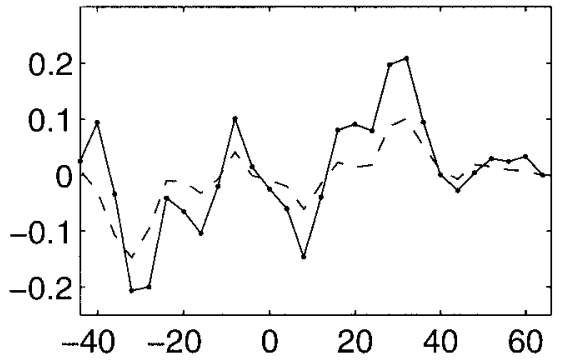

e) Barotropic gyre comp. in the Pacific

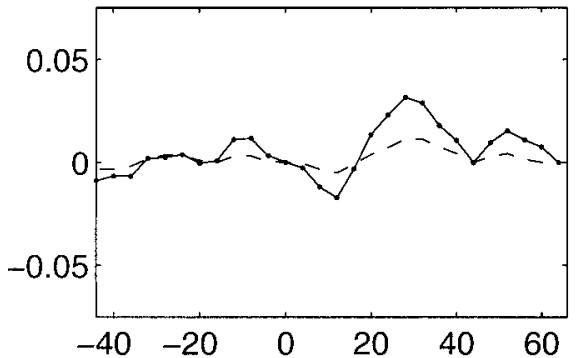

g) Diffusive comp. in the Pacific

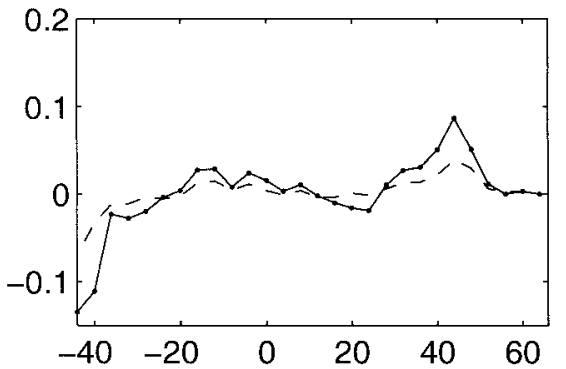

b) Overturning comp. in the Atlantic

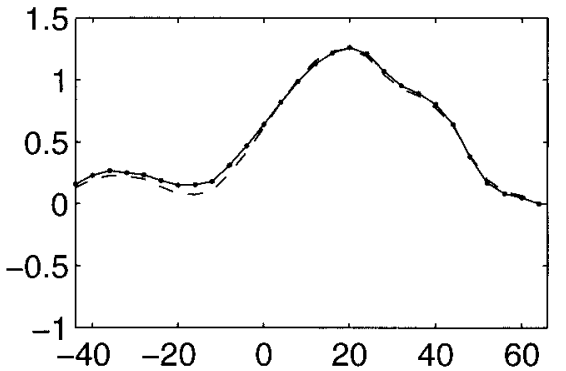

d) Baroclinic gyre comp. in the Atlantic

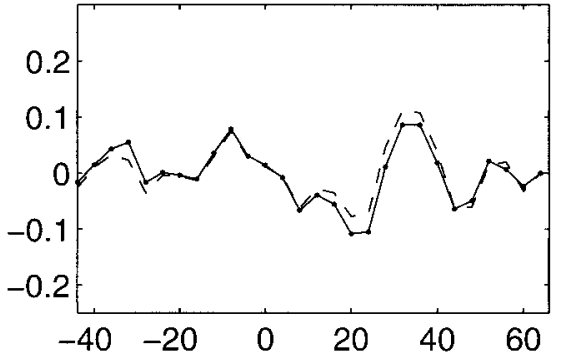

f) Barotropic gyre comp. in the Atlantic

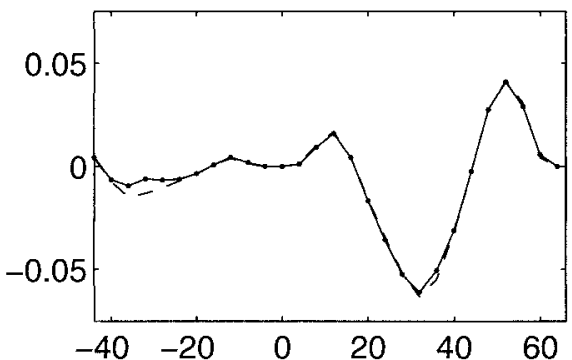

h) Diffusive comp. in the Atlantic

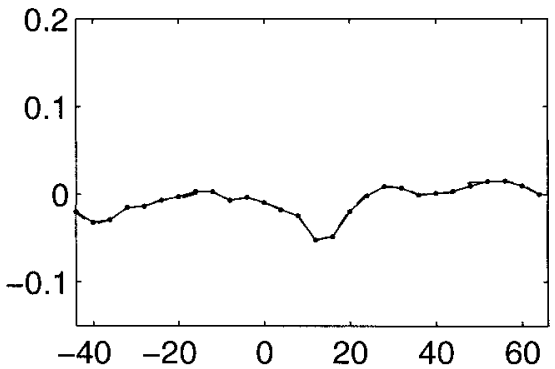

FIG. 18. Comparison of heat transport components in experiment GM (dashed lines), experiment WIDE (solid-dotted lines). Left panels show the Pacific, right panels show the Atlantic. First row: overturning components; second row: baroclinic gyre components; third row: barotropic gyre components; and fourth row: diffusive components. Eddy transports are added to all advective components.

sufficient for reproducing the effects of finer resolution on heat transport.

The results suggest a large effect of the strength and resolution of the western boundary currents on the oceanic heat transport (Wunsch 1996). The representation of the recirculation zones in the Gulf Stream region may prove to be an additional important factor missing in this study. The water particles in these zones spend longer time at the surface, and their density and temperature are strongly affected by the atmospheric fluxes. As a result, the sinking rate in the North Atlantic may change, leading to changes in the heat transport in the basin.

3) We introduce the Gent-McWilliams parameterization in experiment GM and compare the results with experiment FAST. In agreement with some previous studies (Duffy et al. 1997; Weaver and Eby 1997; Jiang et al. 1999, manuscript submitted to J. Phys. Ocean$o g r$.), we find that the meridional overturning weakens as a result of smaller diapycnal mixing in the model. Reduced mixing in the regions away from the North 
Atlantic leads to a decrease in the mass overturning at low latitudes in the North Atlantic (MK), which tends to reduce the overturning component of the heat transport. On the other hand, reduced diapycnal mixing in the North Atlantic allows a larger portion of North Atlantic deep water to arrive at low latitudes (Böning et al. 1995; MK). Hence, introducing the GM scheme produces two competing effects on the low-latitude Atlantic THC, and it is plausible that details of the model configuration and surface forcing determine which of the effects dominates. This would explain why the GM parameterization led to an improvement in THC in many GCMs with more realistic geometry and topography (Danabasoglu et al. 1994; Böning et al. 1995; Jiang et al. 1999, manuscript submitted to J. Phys. Oceanogr.).

In addition, the GM scheme also leads to an increase in the vertical temperature contrast (Danabasoglu et al. 1994; Duffy et al. 1997), which acts to partially compensate for the weakened circulation in the heat transport. The result is a very small change in the heat transport by meridional overturning. This finding is in general agreement with Jiang et al. (1999, manuscript submitted to J. Phys. Oceanogr.), who used the same model as ours but with much more realistic geometry and topography. They reported a large decrease of the overturning from unrealistically high values and a small increase in the heat transport as a result of using the GM scheme. This demonstrates increased efficiency of the zonal mean circulation in transporting heat (same heat transport with weaker mass transport) in a model with the GM parameterization, as opposed to the one with horizontal mixing. An adequate efficiency of the THC in transporting heat is particularly important for the simulation of ocean-atmosphere feedbacks (see Nakamura et al. 1994 and Krasovskiy and Stone 1998). We therefore consider the GM scheme as an overall improvement in the physics of the model despite the small decrease in the heat transport.

4) The basin width is a very important factor for modeling heat transport, as a comparison of the results of the experiments GM and WIDE shows. In the former case, the widths of two basins are the same, whereas in the latter case the Pacific is twice as wide as the Atlantic. Although total uptake of heat does not directly depend on the surface area in our model, the difference between the two experiments is substantial. Significantly larger Pacific heat transport occurs in experiment WIDE, which can be explained by several factors. Larger mass overturning carries more heat in the Southern Hemisphere, whereas a stronger Ekman cell transports more heat at the low latitudes in the Northern Hemisphere. In addition, higher Sverdrup transport leads to a larger mass transport of the western boundary currents, which results in more heat being carried poleward by the horizontal gyres in the model.

In contrast, maximum $\mathrm{THC}$ and heat transport in the Atlantic stay practically unchanged as a result of the widening of the Pacific, although the deep Atlantic out- flow increases significantly. Overall, these results are consistent with MK, who argue, from a detailed analysis of THC dynamics in a single basin spanning both hemispheres, that the amount of cross-equatorial deep flow increases with vertical diffusive transports in the "receiving" portion of the model. Loosely speaking, the Pacific acts like an extension of the South Atlantic here; a wider Pacific enlarges this extension if it mixes uniformly as assumed here.

However, if diapycnal mixing is concentrated near the lateral ocean boundaries, as assumed in Marotzke (1997), Samelson (1998), and MK, widening the Pacific should have no effect on the THC. In reality, rough topographic features lead to enhanced vertical mixing in their vicinity (Polzin et al. 1997), and a wider basin might have more of these features. While there are important questions yet to be addressed, we can conclude that our results point at the important role of basin width in controlling the strength of the THC and heat transport. This is particularly relevant for paleoclimate studies, in which changing basin width may be an important factor in climate changes over periods of millions of years.

The changes added to the configuration of experiment ZONAV (teleconnection between basins, faster boundary currents, improved eddy parameterizations, and more realistic basin widths) were aimed at the improvement of the physics in our idealized model. They resulted in generally increased heat transport where it was originally underestimated, particularly in the South Pacific and at midlatitudes in the Atlantic; see Figs. 6, 9, and 10. Because of the limitations of our model we cannot claim that poor representation of only the physical processes considered here is the main reason for unrealistic latitudinal heat transport in many GCMs. It is plausible that other factors such as a more realistic representation of the ocean's geometry and topography or higher resolution can be equally important for proper simulation of the heat transport in those modes. Nevertheless, our results suggest that by following the steps we have taken, ocean models with intermediate resolution ( $1^{\circ}$ to $2^{\circ}$ in the boundary currents) should be able to accomplish more realistic annual-mean heat transports, thereby coming closer to fulfilling one of the most important criteria for successful coupling with an atmospheric model.

Acknowledgments. This research was funded by the U.S. Department of Energy's (DOE) Office of Biological and Environmental Research and MIT's Joint Program on the Science and Policy of Global Change. Financial support does not constitute an endorsement by DOE of the views expressed in this article.

\section{REFERENCES}

Böning, C. W., W. R. Holland, F. O. Bryan, G. Danabasoglu, and J. C. McWilliams, 1995: An overlooked problem in model simu- 
lations of the thermohaline circulation and heat transport in the Atlantic Ocean. J. Climate, 8, 515-523.

Bryan, F., 1987: Parameter sensitivity of primitive equation general circulation models. J. Phys. Oceanogr., 17, 970-985.

Bryan, K., 1984: Accelerating the convergence to equilibrium of ocean-climate models. J. Phys. Oceanogr., 14, 666-673.

Danabasoglu, G., and J. C. McWilliams, 1995: Sensitivity of the global ocean circulation to parameterizations of mesoscale tracer transports. J. Climate, 8, 2967-2986.

— J. C. McWilliams, and P. R. Gent, 1994: The role of mesoscale tracer transports in the global ocean circulation. Science, 264, $1123-1126$

Duffy, P. B., K. Caldeira, J. Selvaggi, and M. I. Hoffert, 1997: Effects of subgrid-scale mixing parametrizations on simulated distributions of natural $\mathrm{C} 14$, temperature, and salinity in a threedimensional ocean general circulation model. J. Phys. Oceanogr., 27, 498-523.

Fanning, A. F., and A. J. Weaver, 1997: A horizontal resolution and parameter sensitivity study of heat transport in an idealized coupled climate model. J. Climate, 10, 2469-2478.

Gent, P. R., and J. C. McWilliams, 1990: Isopycnal mixing in ocean circulation models. J. Phys. Oceanogr., 20, 150-155.

Gerdes, R., C. Koberle, and J. Willebrand, 1991: The influence of numerical advection schemes on the results of ocean general circulation models. Climate Dyn., 5, 211-226.

Gordon, A. L., 1986: Interocean exchange of thermocline water. $J$. Geophys. Res., 91, 5037-5046.

Haney, R. L., 1971: Surface thermal boundary condition for ocean circulation models. J. Phys. Oceanogr., 1, 241-248.

Hughes, T. M. C., and A. J. Weaver, 1994: Multiple equilibria of an asymmetric two-basin ocean model. J. Phys. Oceanogr., 24, 619-637.

Johns, W. E., T. J. Shay, J. M. Bane, and D. R. Watts, 1995: Gulf Stream structure, transport, and recirculation near $68^{\circ} \mathrm{W}$. J. Geophys. Res., 100, 817-838.

Klinger, B. A., 1996: A kinematic model of wind-driven meridional heat transport. J. Phys. Oceanogr., 26, 131-135.

— and J. Marotzke, 1999: Behavior of double-hemisphere thermohaline flows in a single basin. J. Phys. Oceanogr., 29, 382400 .

Krasovskiy, Y., and P. H. Stone, 1998: Destabilization of the thermohaline circulation by atmospheric transports: An analytic solution. J. Climate, 11, 1803-1811.
Levitus, S., 1982: Climatological Atlas of the World Ocean. NOAA Prof. Paper No. 13, U.S. Dept. of Commerce, NOAA, 173 pp.

Macdonald, A. M., and C. Wunsch, 1996: An estimate of global ocean circulation and heat fluxes. Nature, 382, 436-439.

Marotzke, J., 1997: Boundary mixing and the dynamics of threedimensional thermohaline circulations. J. Phys. Oceanogr., 27, 1713-1728.

— mohaline circulation. J. Phys. Oceanogr., 21, 1372-1385.

— , and P. H. Stone, 1995: Atmospheric transports, the thermohaline circulation, and flux adjustments in a simple coupled model. $J$. Phys. Oceanogr., 25, 1350-1364.

Mikolajewicz, U., T. J. Crowley, A. Schiller, and R. Voss, 1997: Modelling teleconnections between the North Atlantic and North Pacific during the Younger Dryas. Nature, 387, 384-387.

Munk, W. H., 1950: On the wind-driven ocean circulation. J. Meteor., 7, 79-93.

Nakamura, M., P. H. Stone, and J. Marotzke, 1994: Destabilization of the thermohaline circulation by atmospheric eddy transports. J. Climate, 7, 1870-1882.

Polzin, K. L., J. M. Toole, J. R. Ledwell, and R. W. Schmitt, 1997: Spatial variability of turbulent mixing in the abyssal ocean. Science, 276, 93-96.

Samelson, R. M., 1998: Large-scale circulation with locally enhanced vertical mixing. J. Phys. Oceanogr., 28, 712-726.

Tziperman, E., 1997: Inherently unstable climate behavior due to weak thermohaline ocean circulation. Nature, 386, 592-595.

Wang, X., P. H. Stone, and J. Marotzke, 1995: Poleward heat transport in a barotropic ocean model. J. Phys. Oceanogr., 25, 256-265.

,-- , and -1999 : Global thermohaline circulation. Part I: Sensitivity to atmospheric moisture transport. J. Climate, 12, $71-82$.

Weaver, A. J., and T. M. C. Hughes, 1996: On the incompatibility of ocean and atmosphere models and the need for flux adjustments. Climate Dyn., 12, 141-170.

—, and M. Eby, 1997: On the numerical implementation of advection schemes for use in conjunction with various mixing parameterizations in GFDL Ocean Model. J. Phys. Oceanogr., 27, 369-377.

Whitworth, T., and R. G. Peterson, 1985: Volume transport of the Antarctic Circumpolar Current from bottom pressure measurements. J. Phys. Oceanogr., 15, 810-816.

Wunsch, C. W., 1996: The Ocean Circulation Inverse Problem. Cambridge University Press, 442 pp. 